Portland State University

PDXScholar

7-20-2020

\title{
Dynamic Effects of Inertial Particles on the Wake Recovery of a Model Wind Turbine
}

Sarah E. Smith

Portland State University

Follow this and additional works at: https://pdxscholar.library.pdx.edu/open_access_etds

Part of the Aerodynamics and Fluid Mechanics Commons, and the Mechanical Engineering Commons Let us know how access to this document benefits you.

\section{Recommended Citation}

Smith, Sarah E., "Dynamic Effects of Inertial Particles on the Wake Recovery of a Model Wind Turbine" (2020). Dissertations and Theses. Paper 5544.

https://doi.org/10.15760/etd.7418

This Thesis is brought to you for free and open access. It has been accepted for inclusion in Dissertations and Theses by an authorized administrator of PDXScholar. Please contact us if we can make this document more accessible: pdxscholar@pdx.edu. 


\section{Dynamic Effects of Inertial Particles on the Wake Recovery \\ of a Model Wind Turbine}

by

Sarah E. Smith

A thesis submitted in partial fulfillment of the

requirements for the degree of

Master of Science

in

Mechanical Engineering

Thesis Committee:

Raúl Bayoán Cal, Chair

Gerald Recktenwald

Mark Weislogel

Portland State University

2020 


\begin{abstract}
Impacting particles such as rain, dust, and other debris can have devastating structural effects on wind turbines, but little is known about the interaction of such debris within turbine wakes. This study aims to characterize behavior of inertial particles within the turbulent wake of a wind turbine and relative effects on wake recovery. Here a model wind turbine is subjected to varied two-phase inflow conditions, with wind as the carrier fluid $\left(R e_{\lambda}=49-88\right)$ and polydisperse water droplets (26 to $45 \mu \mathrm{m}$ in diameter) at varied concentrations $\left(\Phi_{v}=0.24 \times 10^{-5}-1.3 \times 10^{-5}\right)$, comparing with sub-inertial particles that follow the inflow streamlines. Phase doppler interferometry (PDI) and particle image velocimetry (PIV) were employed at multiple downstream locations, centered with respect to turbine hub height. Analysis considers momentum and particle size distribution within the wake focusing on turbulence statistics and preferential concentration. PDI data show droplet size varied with wake location and volume fraction, and the inflow velocity of $R e_{\lambda}=66.58$ demonstrated $\Phi_{v}$-dependent increases in streamwise velocity deficits of $59.5 \%$ to $62.6 \%$ and $15.8 \%$ to $19.8 \%$ for near and far wake, respectively. PIV data indicated correlation of particle concentration to wake expansion and amplified downward trajectory over the entire interrogation field. Contributions to kinetic energy and momentum are diminished overall for inertial particle cases compared to single-phase, except turbulent momentum flux $\overline{u^{\prime} v^{\prime}}$, where shearing effects are visible at the rotor top edge in near wake and concentrated magnitudes increase in far wake correlating with increased $\Phi_{\nu}$. Application of Voronoi analysis identified clustering behavior in far wake and was validated as motivation for future studies.
\end{abstract}




\section{Acknowledgements}

Martin Obligado, Henda Djeridi, Daniel Mora, Laure Vignal, and all at LEGI for their guidance and welcoming presence.

Mark Weislogel and Gerry Recktenwald for sharing their enthusiasm, expertise, and mentorship.

Raúl Bayoán Cal for seeing my potential before I could, all the high-fives on successes disguised as hurdles, and for the tools to take on turbulence inside the lab and out.

All of my colleagues in the Wind Energy and Turbulence Lab at PSU for sharing their knowledge, time, and support.

My amazing partner and my many versions of family for... everything. 


\section{Table of Contents}

Abstract

Acknowledgements $\quad$ ii

List of Tables $\quad$ iv

List of Figures $\quad$ v

Nomenclature vii

Chapter 1 Introduction 1

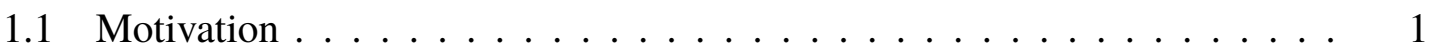

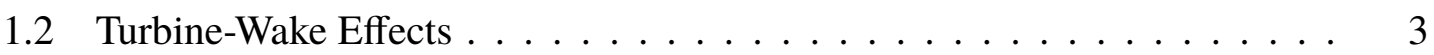

1.3 Debris Impacts . . . . . . . . . . . . . . . . . . . 5

1.4 Particles and Turbulence . . . . . . . . . . . . . . . . . 7

1.5 Present Study . . . . . . . . . . . . . . . . . . . 9

$\begin{array}{lll}\text { Chapter } 2 \text { Theory } & 10\end{array}$

2.1 Turbulent Flows . . . . . . . . . . . . . . . . . . . . . 10

2.2 Multi-phase Flow . . . . . . . . . . . . . . . . . . . . . . 11

2.3 Voronoi Analysis . . . . . . . . . . . . . . . . . . . . . 12

2.4 Present Discussion . . . . . . . . . . . . . . . . . . . 14

Chapter 3 Experimental Setup and Data Acquisition 15

3.1 Experimental Setup . . . . . . . . . . . . . . . . . . . 15

3.2 Hot-wire Anemometry . . . . . . . . . . . . . . . . 17

3.3 Phase Doppler Interferometry . . . . . . . . . . . . . . . . . . . . . . . . . . . . . . . .

3.4 Particle Image Velocimetry . . . . . . . . . . . . . . . . . . 21

Chapter 4 Results 23

4.1 Phase Doppler Interferometry . . . . . . . . . . . . . . . . . . . . . . . . . . 23

4.2 Particle Image Velocimetry . . . . . . . . . . . . . . . . 25

4.3 Voronoi Analysis . . . . . . . . . . . . . . . . 37

$\begin{array}{lll}\text { Chapter } 5 & \text { Conclusions } & 39\end{array}$

Chapter 6 Future Work $\quad 42$

$\begin{array}{ll}\text { Bibliography } & 43\end{array}$ 


\section{List of Tables}

Table 3.1 Experimental cases: All cases represent PIV measurements, in both the near and far wake. Gray cells represent corresponding PDI measurements. . . . . . . . . . . . . . . . 20 


\section{List of Figures}

Figure 1.1 Large-scale wind arrays in varied terrain and conditions: (a)Blue Canyon Wind Farm, Apache, OK [14]; (b) Storm cell above Spinning Spur III wind farm, Adrian, TX [36]; (c) Dust storm over array, Mohave Desert [25] . . . . . . . . . . . . . . . . . . . .

Figure 1.2 Velocity and momentum effects in turbine wakes: (a),(b) Vorticity $\omega$ and vertical turbulent intensity $I_{w}$ in near wake of a model rotor [40]; (c),(d) Vertical profiles of streamwise velocity $U$ and Reynolds shear stress behind a rear, central turbine in a $3 \times 3$ array

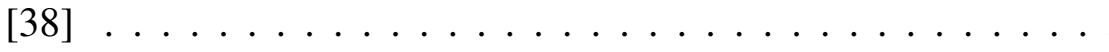

Figure 1.3 Effect of impacting particles on turbine blades: (a) Erosion concentration on blades in reference to angle of impact, particle size, and freestream inflow velocity [16]; (b) Effect of mean particle diameter on turbine blade lifetime; (c) Representations of physical damage on turbine blades by impacting particles [17] . . . . . . . .

Figure 1.4 Numerical simulation results on multi-phase flows: (a) Sweep-stick mechanism in turbulent flow by Homann and Bec. Black dots show particles swept with local velocity, red dots are locations of sticking particles; (b) Contours of mean, normalized particle concentration for the wake of an immobile sphere. Reynolds number increases from top to bottom [22]; (c) Snapshot of particle position planar to axis of symmetry for flow around an immobile sphere [22]. . . . .

Figure 2.1 Voronoi cell distribution example with randomly distributed

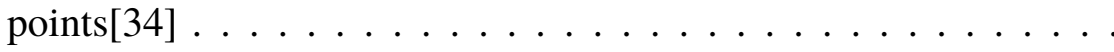

Figure 3.1 Schematic of wind tunnel experimental setup . . . . . . . . . 16

Figure 3.2 Experimental turbine model: (a) Representative schematic. Dotted circle showsspinning rotor projection in $\mathrm{x}-\mathrm{y}$ plane; (b) Image of actual model in tunnel . . . . . . . . . . . . . . 16

Figure 3.3 Power spectral density (PSD) from hot-wire measurements. Normalized PSD $\left(P S D / \sigma_{u}^{2}\right)$ at mean inflow velocities of $U_{\infty}=2.61$, 4.86, and $8.3 \mathrm{~ms}^{-1}$ : (a) Open Tunnel, (b) Near wake of model (1D), and (c) Far wake of model (9.6D); (d) PSD for all three configurations at a single mean velocity $U_{\infty}=4.86 \mathrm{~ms}^{-1} \ldots \ldots \ldots 18$ 
Figure 3.4 PDI-captured particle diameters: (a) PDF of diameter counts. Solid lines represent open-tunnel (i.e., sans turbine). Top two subfigures show far wake (9.6D) for water injection flow rates 1.7 and $2 \mathrm{~L} / \mathrm{min}$ (left and right). Lower plots show near wake (1D); (b) Mean diameter bar plot for varied injection rates, wake location, and model presence. Slanted stripes denote injection of $1.7 \mathrm{~L} / \mathrm{min}$, horizontal are near wake locations, colored bars represent model present, and white bars open-tunnel. . . . . . . . . . . . . . . .

Figure 4.1 PDF of count ratio for instantaneous streamwise velocity within the turbine wake. Solid lines represent velocity distributions of opentunnel configuration (no turbine present). Top row subfigures, left and right, represent data collection at the far wake location (9.6D) for a uniform distribution of water injected at flow rates 1.7 and $2 \mathrm{~L} / \mathrm{min}$ respectively from the spray grid. Bottom row subfigures represent data collected within the rotor near wake $(1 D)$ at each of the respective flow rates . . . . . . . . . . . . .

Figure 4.2 PDF of count ratio for instantaneous streamwise velocity within the turbine wake for $R e_{\lambda}=66.58$. Solid lines represent velocity distributions of open-tunnel configuration (no turbine present). . . . . .

Figure 4.3 Planar wake contours of normalized streamwise velocity deficits $\left(U_{\infty}-U\right) / U_{\infty}$ for three values of Taylor-based Reynolds number $\left(R e_{\lambda}\right)$ increasing from left to right, and four volume fractions $\left(\Phi_{v}\right)$ increasing in rows from top to bottom. ...........

Figure 4.4 Near wake, normalized mean statistics. Left to right: Streamwise velocity $U / U_{\infty}$; Vertical velocity $V / U_{\infty}$; Reynolds Stresses $\overline{u^{\prime} u^{\prime}} / U_{\infty}^{2} ; \overline{u^{\prime} v^{\prime}} / U_{\infty}^{2} ; \overline{v^{\prime} v^{\prime}} / U_{\infty}^{2} \ldots \ldots \ldots \ldots \ldots \ldots$

Figure 4.5 Far wake, normalized mean statistics. Left to right: Streamwise velocity $U / U_{\infty}$; Vertical velocity $V / U_{\infty}$; Reynolds Stresses $\overline{u^{\prime} u^{\prime}} / U_{\infty}^{2} ; \overline{u^{\prime} v^{\prime}} / U_{\infty}^{2} ; \overline{v^{\prime} v^{\prime}} / U_{\infty}^{2} \ldots \ldots \ldots \ldots \ldots \ldots$

Figure 4.6 Vertical profiles of normalized, time-averaged statistics, spatially averaged over $1 \mathrm{~cm}$ within near and far wake of the turbine model $(1 D$ and 9.6D). Dotted lines represent far wake locations. Left to right: Mean streamwise velocity (U); vertical velocity (V); and standard deviation $\left(\sigma_{u}\right)$; Reynolds stresses $\left.\left.\left\langle\overline{u^{\prime} u^{\prime}}\right\rangle ; \overline{u^{\prime} v^{\prime}}\right\rangle ; \overline{\nu^{\prime} v^{\prime}}\right\rangle$.

Figure 4.7 Voronoi analysis images for $R e_{\lambda}=66.58, \Phi_{\nu}=\Phi_{1.7}$ : (a) Sample PIV image used for particle detection, interrogation area outlined in red; (b) Voronoi cell image for a single image; (c) PDF of normalized cell area $b=S /\langle S\rangle$ plotted against random Poisson process distribution. . . . . . . . . . . . . . . . . . . . 


\section{Nomenclature}

$\lambda \quad$ Taylor microscale, see equation (2.2), page 11

$\langle\ldots\rangle \quad$ Quantity spatial averaging

$v \quad$ Fluid kinematic viscosity

$\bar{u} \quad$ Time-averaged fluid velocity

$\Phi_{v} \quad$ Particle-Fluid volume fraction

$\rho_{f}, \rho_{p}$ Fluid, particle density

$\sigma \quad$ Standard deviation

$\tau_{\eta} \quad$ Kolmogorov time scale

$\tau_{p} \quad$ Particle viscous relaxation time

$\varepsilon \quad$ Turbulent dissipation rate, page 11

$b \quad$ Normalized Voronoi cell size

$D \quad$ Diameter of turbine rotor

$f \quad$ Forcing term

$p \quad$ Fluid pressure

$R e_{\lambda} \quad$ Taylor based Reynolds number, page 14

St Stokes number, see equation (2.3), page 11

$u \quad$ Instantaneous fluid velocity

$u^{\prime} \quad$ Fluid velocity fluctuation

$U_{\infty} \quad$ Freestream fluid velocity

S Voronoi cell area 


\section{Chapter 1}

\section{Introduction}

\subsection{Motivation}

Renewable resources have become leading contributors to energy generation capacity throughout the world. In the last several years, power generation from renewable energy has more than doubled globally, with wind energy contributing to an average of $50 \%$ of all renewable energy generation [1]. In 2019, the United States (U.S.) had a total operating wind power capacity of 105,583 MW [2]. Considering the annual consumption of 10,972 $\mathrm{kWh}$ for an average household in 2018, generation on this scale is capable of supplying over 84 million homes with electricity per year [3]. While this bodes well for the future of wind power, consideration must be taken in terms of environmental conditions to optimize power output and ensure turbine longevity. Most of the U.S. wind harvesting systems are located in the west and midwest regions of the country, with the top five capacity states making up more than $56 \%$ of the annual tally - Texas, Iowa, Oklahoma, Kansas, and California. These states are known for their expansive and open ranges, making them optimal locations for maximum turbine interaction with unimpeded, high velocity winds. On a day with clear skies and high winds, power generation is at peak efficiency for locations such as Blue Canyon wind farm in Figure 1.1(A). The expansive nature of such regions also means they are vulnerable to sudden changes in weather patterns and seasonal storms, especially in tornado season within the central plains (Figure 1.1(B)), where bursts of high winds hurl large quantities of rain, hail, and other debris over vast distances. Even in the absence of 
storms, less ideal locations with loose soil like the Mohave Desert (Figure 1.1(C)) are subjected to repeated impacts of sand, small rocks, and other dense particles carried in high winds which cause devastating destruction to turbines and turbine blades over time.

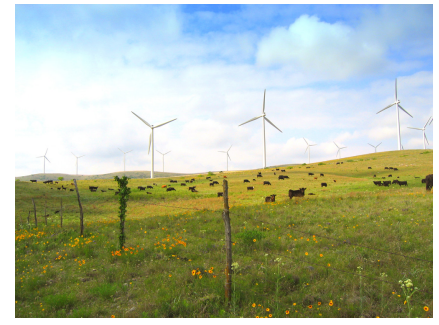

(A)

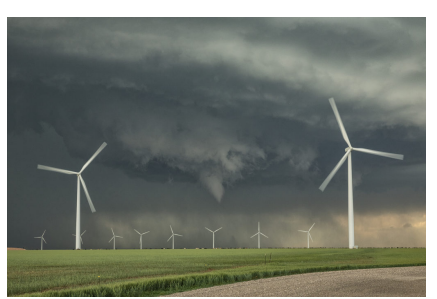

(B)

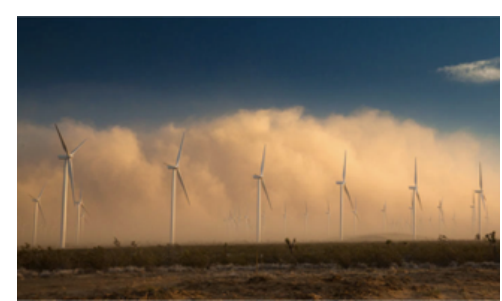

(C)

Figure 1.1: Large-scale wind arrays in varied terrain and conditions: (a)Blue Canyon Wind Farm, Apache, OK [14]; (b) Storm cell above Spinning Spur III wind farm, Adrian, TX [36];

(c) Dust storm over array, Mohave Desert [25].

In addition to the damage particles such as dust, rain and other debris impose on wind farm equipment, they also can alter what would normally be considered steady flow within the array increasing the complexity within an already turbulent regime $[6,24]$. Turbulent boundary layers as inhabited by large-scale wind plants significantly affect power efficiency and wake propagation over the entire system. Considering wind as a gas phase carrier fluid, the addition of solid or liquid debris into the flow introduces complications in the form of multi-phase flow dynamics. The difference in density and finite size of these particles from that of the carrier flow provokes physical interactions between the two phases, behaving as inertial particles rather than what are considered massless tracers like smoke. This study introduces discussion of particle-wake interaction within turbine wakes, giving an initial understanding without the complicating influence of boundary effects and aims to discuss the interaction of debris as inertial particles within the turbulent wake of a model wind turbine subjected multi-phase flow. Largely important in this work is the noted wake persistence due to inertial particle presence, identified locations and features of particle entrainment, and the size and distribution of particles within the turbine wake. 


\subsection{Turbine-Wake Effects}

Even when considering the most ideal atmospheric conditions with fully-developed inflow, turbulence propagation within wind energy harvesting arrays disrupts optimal wind flow conditions and can provoke devastating effects on energy production capabilities beyond the first row of turbines $[21,20]$. Such disturbances are the product of rotor-flow interactions at the first set of rotors, diminishing streamwise velocity across the rotor shadow and producing vortices along the rotor edge of the wake directly after the interaction takes place (Figures 1.2(B), 1.2(A)) [40].
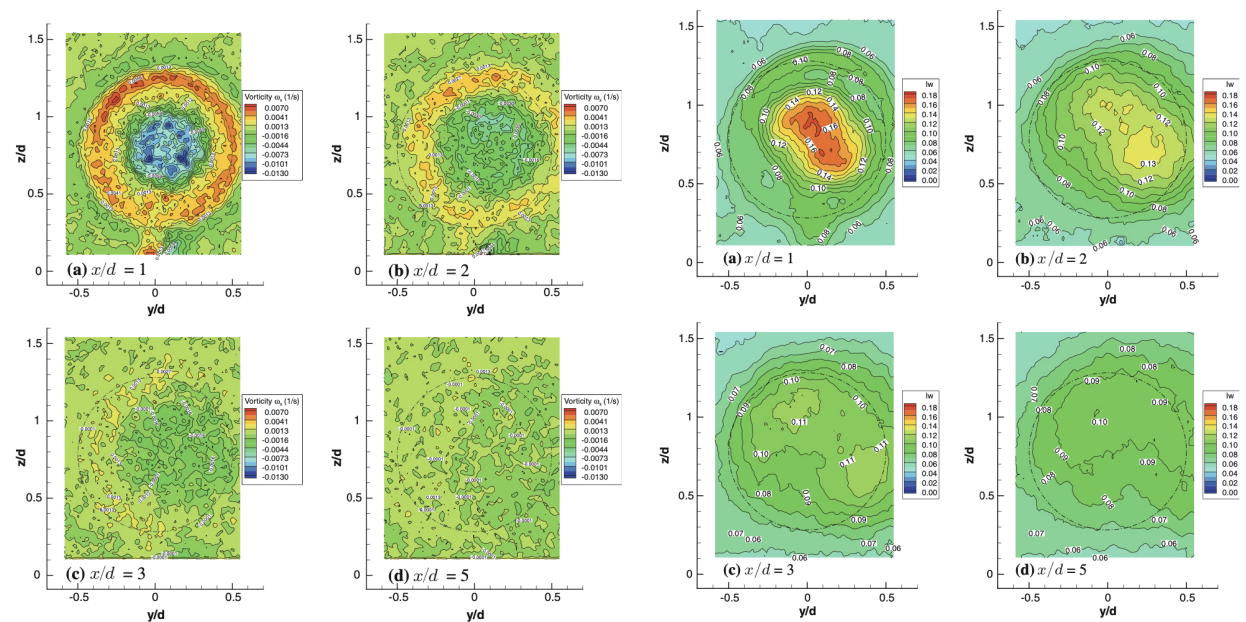

(A)

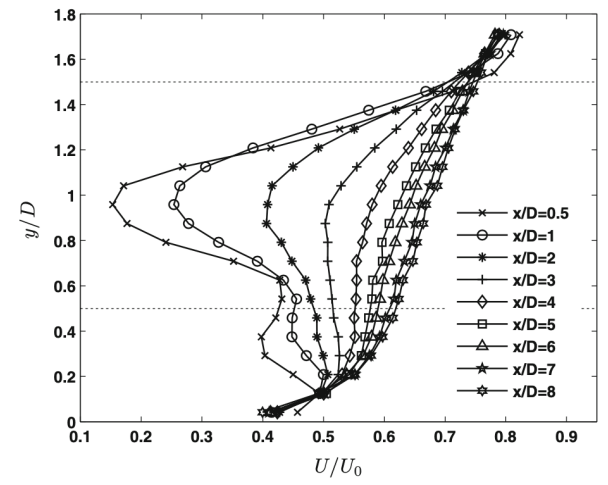

(B)

(C)

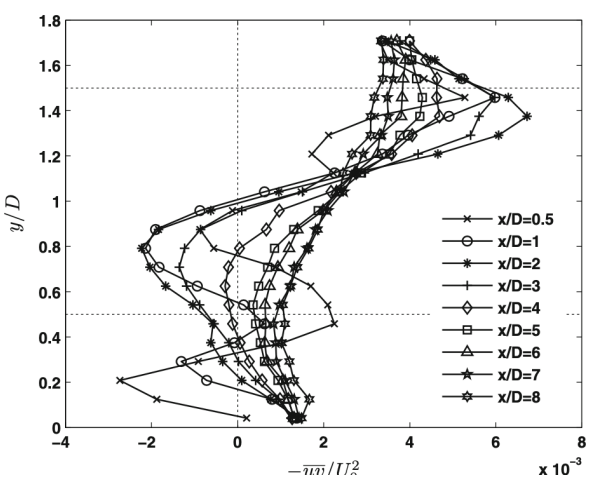

(D)

Figure 1.2: Velocity and momentum effects in turbine wakes: (a),(b) Vorticity $\omega$ and vertical turbulent intensity $I_{w}$ in near wake of a model rotor [40]; (c),(d) Vertical profiles of streamwise velocity $U$ and Reynolds shear stress behind a rear, central turbine in a $3 \times 3$ array [38] 
The wake regions expand downstream until interaction with the outer flow eventually recovers the wind flow to a state similar to the unimpeded wind upstream of the turbine. Changes in flow trajectory and velocity degradation are signals of beginning wake recovery, but these cues are only portions of the process toward regaining a fully developed flow state. Turbine wakes are observed to have significant effects on energy content of a flow, producing low velocities in the rotor shadow bordered by highly vortical regions, which persist significant distances downstream of single and arrayed turbine configurations [8, $20,7,37,38]$. One set of field experiments on a single turbine found velocity deficits of $25 \%$ comparing the inflow velocity to $6 D$ downstream, coupled by an increase in downward turbulent momentum transport [33]. For a full-size turbine, this compares to 600 meters behind the rotor. Wind velocity deficits imposed by the turbine have been shown around $80 \%$ less than the inflow at $0.5 \mathrm{D}$, with recovery starting between 5 and $8 D$ (Figures $1.2(\mathrm{C})$, 1.2(D)) [38]. Chamorro and Porté-Agel reported large increases in turbulence intensity along the upper part of the wake of a single turbine, most visible along the rotor edge of the wake, where strong shearing and helicoidal vortices are present. Velocity deficits and turbulence intensity were found to be nearly recovered only after $15 D$ downstream [8]. The persistence and intensity of these vortical structures have been examined as in the work of Odemark and Fransson, whose turbine model was inspiration for the one used in the present study [31]. They found the vortical strength to diminish greatly at a distance of $2.5 \mathrm{D}$ downstream of the turbine model, showing no sign of the effect after $4 D$. Since most wind farm arrays are situated only $5 D$ to $10 D$ apart in the downstream direction, this suggests that only the first turbine row is encountering ideal conditions while the rest of the farm is subject to less predictable flow states. 


\subsection{Debris Impacts}

These studies speak to conditions within the wind plant in relatively stable conditions, but neglect the case of turbines subjected to unimpeded winds laden with debris such as rain, dust, and other dense materials. Numerous researchers have worked to quantify the effects of debris presence and impingement finding significant changes in productivity and longevity of wind turbines and turbine blades [23, 39, 24, 17, 12, 9, 6]. Corrigan and DeMiglio performed one of the first field experiments to identify rainfall-induced performance degradation [10]. Their work cited performance decreases between approximately 18-27\% depending on respective levels of rainfall rates for a single turbine configuration, also noting that increased wind speeds and blade angle of attack further promote the adverse effects of precipitation. Researchers have since examined possible reasons for this decrease in power including changes to blade surface roughness due to water film creation on the surface and have expanded to changes in geometry due to the accumulation on the blade by dust, ice and other debris $[6,23]$. Cohan and Arastoopour performed computational fluid dynamics (CFD) simulations to model a turbine blade as an airfoil showing that increased rainfall rates accompany a decrease in airfoil performance for relatively low rainfall rates (i.e., volume fractions), lowering the airfoil lift coefficient and raising the drag. There exists a limit to this trend where heavy rainfall rates greater than $120 \mathrm{~mm} / \mathrm{hr}$ showed increases in lift coefficients, but still produced higher drag than in dry, single-phase flow conditions [9].

Wind harvesting plants located in dry environments are more prone to dust and other solid debris impinging and accumulating on turbines. Khalfallah and Koliub showed that dust accumulation alone can alter the surface roughness of turbine blades and provoke losses in power production. Collecting dust particle size and area of accumulation, they calculated losses of $6.5-38 \%$ for accumulated particle diameters ranging from $50-300 \mu \mathrm{m}$ [23]. Surface accumulation of particles degrades performance and is costly to alleviate, 
but is a relatively temporary effect of debris interaction with turbine blades compared to the damage inflicted by repeated particle impacts in heavy winds. Fiore and Selig created a simulation model to study the relationship of turbine blade erosion and lifespan to turbine configurations and dust diameter. Their results highlighted the effect of particle size, impact angle, and carrier velocity on the location and propensity of material erosion (Figure 1.3(A)) [16]. They further compared their results with blade erosion on actual field turbine blades (Figure 1.3(C)) and identified three phases of blade degradation, finding that increases in grain diameter directly related to a decrease in lifespan (Figures 1.3(B)) [17].
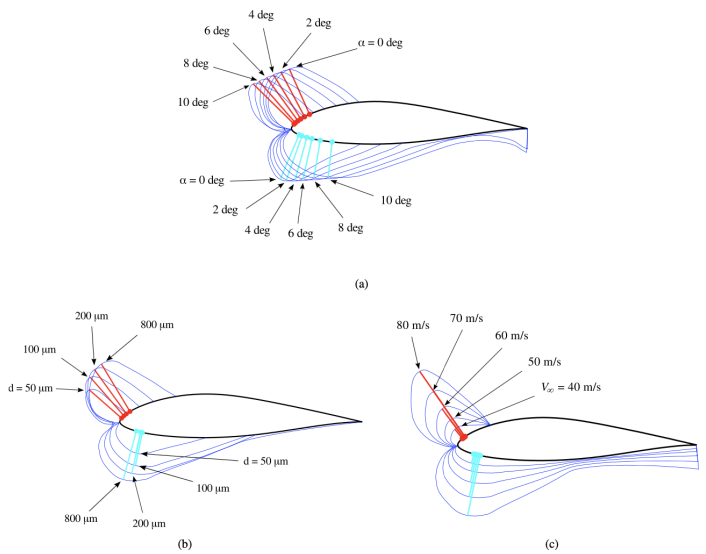

(A)
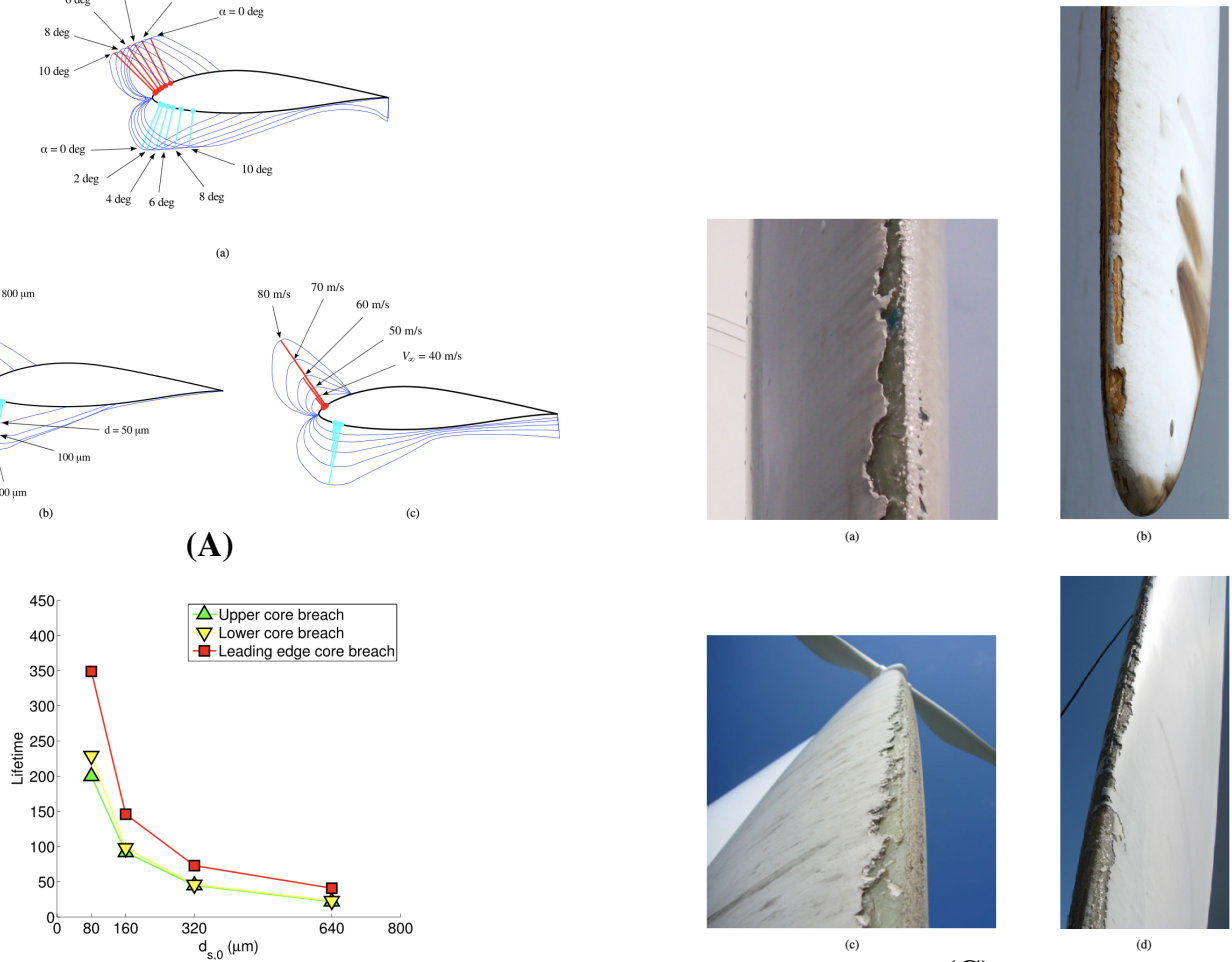

(B)
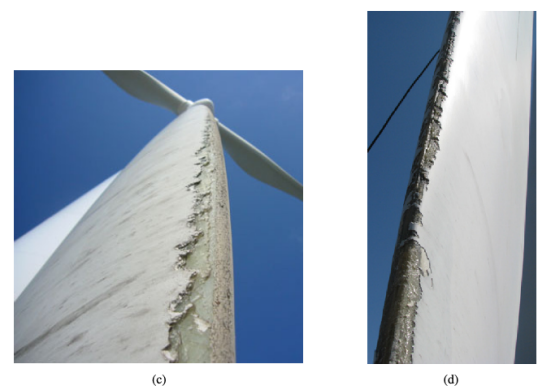

(C)

Figure 1.3: Effect of impacting particles on turbine blades: (a) Erosion concentration on blades in reference to angle of impact, particle size, and freestream inflow velocity [16]; (b) Effect of mean particle diameter on turbine blade lifetime; (c) Representations of physical damage on turbine blades by impacting particles [17]

Li et al. found that turbine blade erosion is more sensitive to changes in Stokes number than to blade angle of attack, citing that erosion occurs even at a relatively small values 
$(S t>0.015)$. Stokes number is defined as the ratio of particle relaxation within a flow $\left(\tau_{p}\right)$ to the Kolmogorov time scale $\left(\tau_{\eta}\right)$ of the carrier flow. Varying parameters such as wind velocity and angle of attack showed little effect on level of blade erosion when Stokes number was held at 0.15 [24].

\subsection{Particles and Turbulence}

The aforementioned studies address wake effects of individual and multi-unit wind plants in single-phase flow, and separately of the degrading impacts of rain, entrained dust, insects, and other debris impinging on a single turbine. But the question remains as to how the presence of such particles affect turbine wake behavior and how wake turbulence may alter their behavior and trajectories. As previously noted, the vast difference in density between wind and denser debris such as rain and granular object suggests the interaction is a multi-phase phenomenon, gaining insight here from research describing particle behavior and effects within turbulent flows $[28,18,22,30]$. Studies toward particle behavior in a variety of turbulent flows show that in homogenous turbulence, particles of smaller size tend to become entrained in vortices, while larger particles are propelled away by large scale features [13]. These collections of particles (i.e., clusters) exhibit preferential concentrations and can collectively affect the behavior and energy content of the surrounding fluid. Discussion of this mechanism has since been complemented as having a 'sweep-stick' behavior, describing particle tendency to follow the local fluid velocity (sweep) and adhere to structures within the flow (stick) dependent on the particle size and ultimately the particle relaxation time (Figure 1.4(A)) [19]. Many methods exist to identify and characterize the locations and behaviors of particle clustering within turbulent flow (cf. an extended review by Monchaux, Bourgoin and Cartellier)[28]. Significant here is the method of Voronoi diagram distributions which identify cluster and void concentration within a spatial context. 


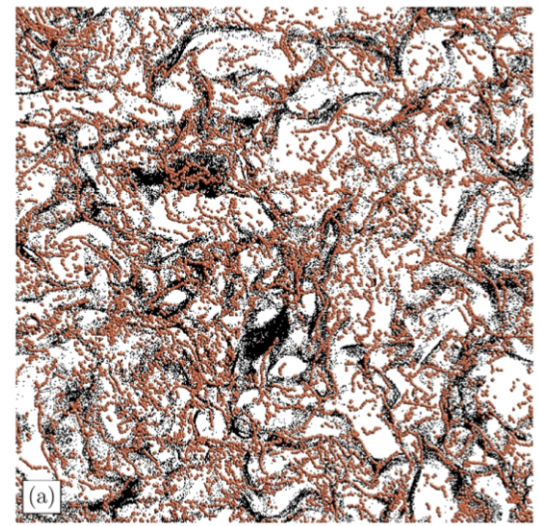

(A)
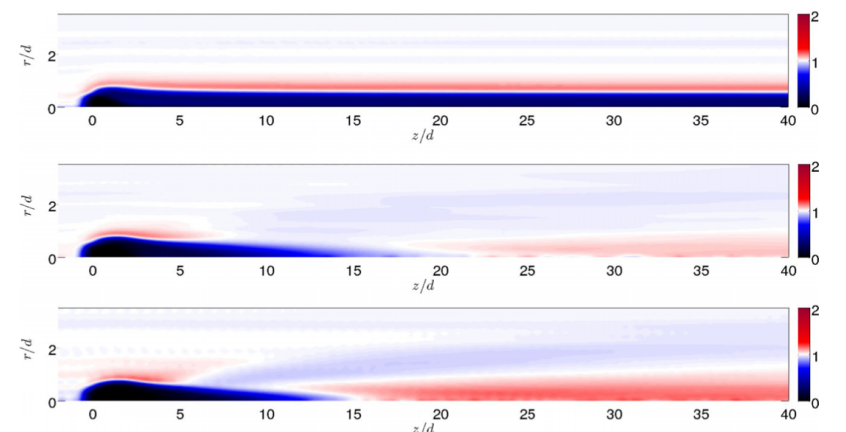

(B)

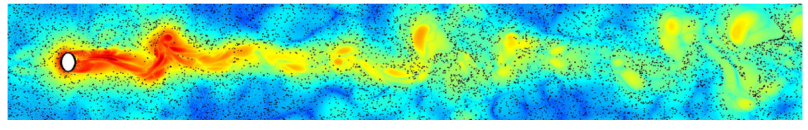

(C)

Figure 1.4: Numerical simulation results on multi-phase flows: (a) Sweep-stick mechanism in turbulent flow by Homann and Bec. Black dots show particles swept with local velocity, red dots are locations of sticking particles; (b) Contours of mean, normalized particle concentration for the wake of an immobile sphere. Reynolds number increases from top to bottom [22]; (c) Snapshot of particle position planar to axis of symmetry for flow around an immobile sphere [22].

Clustering has been found to be most prevalent when the response time of the particle is on the order of the Kolmogorov scale within the dissipative range and has been found to affect particle acceleration especially within vortical structures [4]. In terms of the turbine wake, this could affect the turbulence properties as found along the rotor edge [8]. Sumbekova et al. explored the effect of Reynolds number $\left(R e_{\lambda}\right)$ and volume fraction $\left(\Phi_{v}\right)$ on sub-Kolmogorov scale particle clustering within two-phase, homogeneous isotropic turbulent flow. Their work showed increases in cluster presence when either Reynolds $R e_{\lambda}$ or $\Phi_{v}$ were increased, with a primary dependence of cluster and void size on $R e_{\lambda}$ [34]. Numerical simulations by Homann and Bec examined the wake of an immobile sphere subjected to two-phase turbulent flow, showing preferential concentrations of particles with small to moderate inertia on the outer edges of the wake, with lesser populations in the wake center. These regions also contained vortical structures characterized by particle clusters surrounding void regions (Figure 1.4(C)). For low Reynolds numbers, this wake shadow of high concentration persists beyond 40 sphere diameters. However, at higher Reynolds 
numbers, increased concentration focuses throughout the wake profile rather than along the edges, beginning as near as 15 sphere diameters (Figure 1.4(B))[22]. Given the preference of particle concentration in rotating regions this suggests that, at least for blunt objects, particle distribution and acceleration throughout the wake may be a complicating factor for wake recovery $[35,4]$.

\subsection{Present Study}

This study aims to give introductory insight into the complex problem of wind turbines subjected to debris-filled flow fields, by examining the near and far wake of a model turbine within turbulent, homogeneous, isotropic, polydispersed multi-phase flow conditions. While previous studies have approached the subjects of turbulence propagation in turbine wakes and behavior of particles-turbulence wake interactions separately, this is a unique experiment toward understanding of wake characteristics and recovery of a turbine subjected to particle-laden flow.

Single-phase flow statistics at several inflow wind velocities were calculated from hot wire anemometry (HWA) data and particle image velocimetry (PIV) measurements on subinertial tracers which follow the flow to characterize parameters without the presence of inertial particles. Subsequent measurements varying carrier flow (wind) velocity and particle concentration (water) were collected via phase doppler interferometry (PDI) and particle image velocimetry (PIV) to gather and calculate velocity and turbulence parameters within imposed multi-phase flow. Lastly, PIV data were used toward elementary Voronoi analysis to identify clustering effects within the turbine wake. Although full Voronoi analysis is outside the scope of the present discussion, results presented here are intended to inform and motivate future work. Turbulent effects within near and far wakes were examined to gain insight on wake behavior and recovery, particle size and distribution, and gain an introductory understanding of particle concentration and clustering within the turbine wake. 


\section{Chapter 2}

\section{Theory}

\subsection{Turbulent Flows}

Analysis here is characterized from the Reynolds Averaged Navier-Stokes (RANS) equation generalized for incompressible and stationary boundary layer flows

$$
\overline{u_{j}} \frac{\partial \overline{u_{i}}}{\partial x_{j}}=\frac{1}{\rho} \frac{\partial \bar{p}}{\partial x_{i}}-\frac{\partial}{\partial x_{j}}\left(\overline{u_{i}^{\prime} u_{j}^{\prime}}\right)+f_{i},
$$

where $u$ is the fluid velocity, $p$ is pressure, $\rho$ is the carrier fluid density, and $f_{i}$ represents forcing on the carrier fluid imposed by both turbine and particle interactions.

Within this equation, time-averaged quantities are indicated by overbars and fluctuations by prime symbols. For example, instantaneous velocity $u$ is defined as $u=\bar{u}+u^{\prime}$. Subscripts $i$ and $j$ represent the directions 1, 2, 3 within three dimensional space, defined as $\left(x_{1}, x_{2}, x_{3}\right)=(x, y, z)$ representing the respective streamwise, vertical, and spanwise directions. Respective velocity components are defined as $\left(u_{1}, u_{2}, u_{3}\right)=(u, v, w)$. In the present case, all measurements were taken sufficiently far from the wall and the viscous stresses are not considered to affect the mean flow. The total stress is thus dominated by the Reynolds stress term $\partial \overline{u_{i}^{\prime} u_{j}^{\prime}} / \partial x_{j}$ representing momentum flux within the flow.

The Taylor microscale represents the length scale of structures within a turbulent flow at which the energy begins to dissipate and is defined as 


$$
\lambda=\sqrt{\frac{15 v \sigma_{u}^{2}}{\varepsilon}}
$$

where $v$ is the fluid kinematic viscosity and $\sigma_{u}$ is the standard deviation of streamwise velocity. The turbulent dissipation rate is $\varepsilon$ and was calculated from the small-scale modelling of 1-D power spectrum obtained through hot-wire anemometry as $\varepsilon=\int 15 v k_{1}^{2} E_{11} d k_{1}$ where $E_{11}$ is the power spectral density, and $k_{1}$ is the wave number obtained from Taylor hypothesis [29]. The expression for $\varepsilon$ has been shown valid for homogeneous isotropic turbulence as well as axisymmetric wakes [11].

\subsection{Multi-phase Flow}

In general practice, multi-phase flows are characterized by the Stokes number which describes the relationship between the particle viscous relaxation time $\left(\tau_{p}\right)$ and the Kolmogorov time scale $\left(\tau_{\eta}\right)$ for a point particle as

$$
S t=\frac{\tau_{p}}{\tau_{\eta}}=\frac{\rho_{p} d_{p}^{2} \varepsilon^{1 / 2}}{18 \rho_{f} v^{3 / 2}},
$$

where $\rho_{p}$ and $d_{p}$ are the average particle density and diameter and $\rho_{f}$ is the density of the carrier fluid. $S t$ is expressed to vary with the dissipation rate and particle diameter. For point particles as used in this study, the particle diameter is smaller than Kolmogorov scale. The relationship of $S t$ to particle clustering is an active point of current research. One approach is that Stokes numbers near unity $(S t \sim 1)$ predominantly occupy regions between vortices, while those at very small Stokes numbers can be found within vortex structures [28]. Such behavior gives insight to entrainment, where ambient flow is captured by large-scale structures of a turbulent wake and increases energy in the region [26]. However, this mechanism has been found to imply a weak dependence on $S t$ in terms of clustering [34]. The matter of entrainment will not be approached with detailed analysis here, but the underlying mechanisms guide discussion of energy content and particle be- 
havior within turbine wake regions. Another method of describing clustering dependence on $S t$ refers to the relationship between $\tau_{p}$ and $\lambda$ by defining a contraction rate $\gamma$ to the particle relaxation time for a given direction within flow, $\gamma=\lambda \tau_{p}$ [19]. If a collection of very small particles contracts slowly (ie. $\tau_{p} \ll \gamma^{-1}$ ) then their behavior follows that of the fluid and avoid contraction yet cluster around structures. In contrast, for larger particles

with high contraction rates $\left(\tau_{p} \gg \gamma^{-1}\right)$, the relative inertia causes them to bypass structure regions. Most prevalent cluster formation exists when $\tau_{p} \sim \gamma^{-1}$ or when $\tau_{p} \approx \lambda^{-1 / 2}$. This introduces a limit on particle size for clustering behavior based on the largest dissipative scale within a flow.

\subsection{Voronoi Analysis}

Implementation of Voronoi analysis allows for qualitative and statistical characterization of spatial relationships between particles in multi-phase flow. This process can be applied to one, two, or three-dimensional (1-D, 2-D or 3-D) spaces, separating a region into polygonal cells, each enclosing some defined point. Point locations can be described in a given spatial region as randomly distributed, and enclosing cells are created according to the proximity of one point to another as in Figure 2.1.

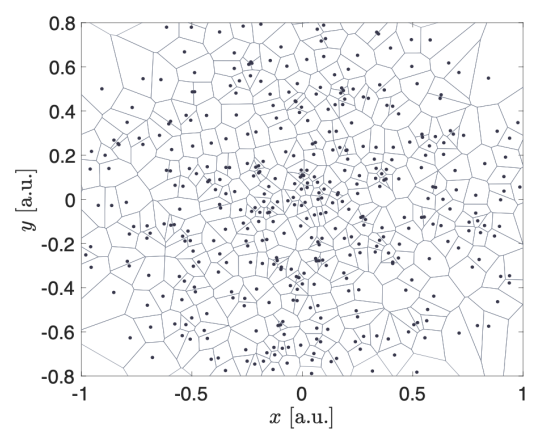

Figure 2.1: Voronoi cell distribution example with randomly distributed points[34]

Data used for this purpose consists of 2-D images. The cell creation method used here is referred to as the 'perpendicular bisector method', where the size and shape of the cells 
are dependent on proximity of individual particles with their surrounding neighbors $[15$, $27,30,34]$. The walls of the polygon are defined as lines (or surfaces in 3-D framework) which are orthogonal to, and located at the midpoint of a straight line from one particle to the next. Smaller cells represent shorter distances between particles, and a collection of smaller cells near to each other is considered to have high local particle concentration, referred to as a cluster. Larger cells are the result of increased distance between neighboring particles and represent regions of low concentration known as voids. When coupled with Stokes analysis, this gives a way to determine the presence and location of structures within a wake.

The level of Voronoi analysis presented for this study is primarily to identify whether the method is suitable for turbine wake applications and present motivation for future work. Statistical comparison to gauge this is based on the work of Monchaux et al., discussing the behavior of Voronoi distributions through use of Poisson Voronoi diagrams (PVD) [27]. The concept suggests that if the Voronoi centers are randomly distributed and uncorrelated within a space, the cell regions can be modelled analytically using a Poisson distribution. For a given 2-D space, the normalized distribution takes the form of

$$
f_{2-D}(b)=\frac{343}{15} \sqrt{\frac{7}{2 \pi}} b^{5 / 2} \exp \left(-\frac{7}{2} b\right)
$$

where $b$ is the normalized cell size based on cell area $S(b=S /\langle S\rangle)$ [27]. In contrast to the Poisson process, the Voronoi cell centers for multi-phase turbulent flows take on non-random distributions as the particles surround or become entrained in coherent flow structures. Therefore, the probability distribution must differ from the above form and the standard deviation of cell regions in actual flows will be greater than that for a random Poisson process (i.e. $\sigma_{R P P}<\sigma_{\text {voro }}$ ) where for this distribution $\sigma_{R P P}=0.53$. 


\subsection{Present Discussion}

The present study considers polydisperse flow, meaning the flow contains similarly shaped particles with size variations rather than particles of identical shape and size. Capturing $S t$ within all regions considered here requires higher spatial and temporal resolution than used to collect data in the present study. As such, average droplet size quantities are observed toward initial understanding of entrainment. Additionally, each case is referenced with regard to the Taylor-based Reynolds number $\left(R e_{\lambda}=\sigma_{u} \lambda / v\right)$ which points to the ratio between inertial and viscous forces for a given flow based on its single phase counterpart, and particle volume fraction $\left(\Phi_{v}=V_{p} / V_{f}\right)$ describing the ratio of the volume inhabited by particles within the flow space to that of the carrier fluid (air). 


\section{Chapter 3}

\section{Experimental Setup and Data Acquisition}

\subsection{Experimental Setup}

Experiments were conducted in the Lespinard wind tunnel at Laboratoire des Écoulements Géophysiques et Industriels (LEGI), Université Grenoble Alpes (Grenoble, France). This closed-loop tunnel has a $4 \mathrm{~m}$ long test section, with cross-sectional dimensions of $0.75 \mathrm{~m} \times$ $0.75 \mathrm{~m}$ as shown in Figure 3.1. A passive grid was situated at the tunnel inlet followed by an additional passive spray grid, producing mild turbulence on the order of 2.4-3.1\% from the tunnel entrance as calculated from hot-wire anemometry data and is discussed further in a later section. Each linear portion of the spray grid held a misting nozzle with outlet diameter of $0.4 \mathrm{~mm}$, producing a uniform distribution of polydispersed water droplets with particle diameters ranging from 18 to $40 \mu \mathrm{m}$ as determined from the maximum probability expressed for all cases in Figure 3.4(A). Water was supplied to the spray grid via high pressure pump (Hawk NDH8515) capable of pressure at 150 bar and a volumetric flow rate of $8 \mathrm{~L} / \mathrm{min}$. Water flow rate was controlled manually using a variable regulator connected to the pump. 


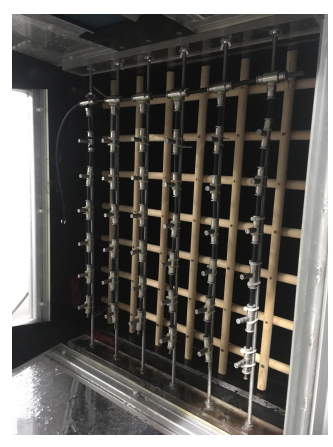

(A)

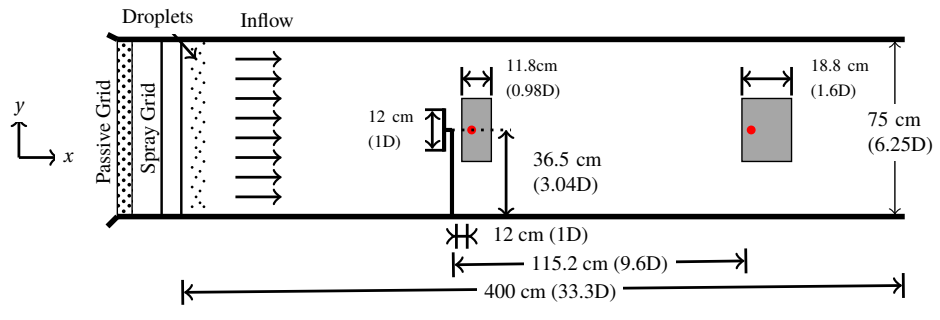

(B)

Figure 3.1: Schematic of wind tunnel experimental setup, side view. Grey boxes indicate twodimensional PIV planes and red dots represent PDI and hotwire anemometry locations for data acquisition. (Schematic is not to scale.)

The model used for this work was a three-blade, horizontal axis turbine whose rotor was based on the design of Odemark and Fransson, employing a Blade Element Momentum (BEM) scheme with Glauert optimization $[31,5]$. The rotor blade pitch was held at $6.5^{\circ}$ to agree with Odemark and Fransson, and rotor diameter was modified to be $12 \mathrm{~cm}$, representing experimental scaling of 0.001:1 compared to full scale turbines (Figure 3.2).

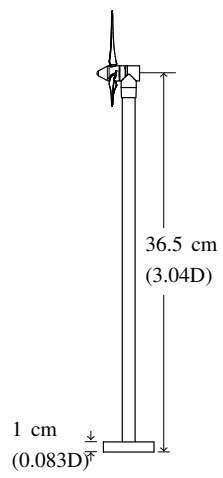

(A)

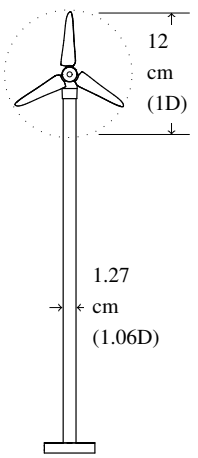

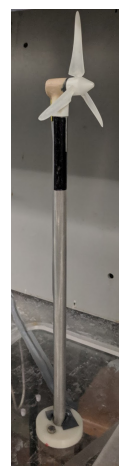

(B)

Figure 3.2: Experimental turbine model: (a) Representative schematic. Dotted circle showsspinning rotor projection in $x-y$ plane; (b) Image of actual model in tunnel

Data were collected within the open-tunnel (no model) and at varied locations within the downstream wake of the turbine using hot-wire anemometry, phase doppler interferometry (PDI), and particle image velocimetry (PIV) as discussed in the following sections. The model was centrally positioned within the tunnel cross-section with the nacelle a ver- 
tical distance of $36.5 \mathrm{~cm}$ from the tunnel floor. A minimum spanwise distance of 36.5 $\mathrm{cm}$ for the PDI and hot-wire measurements, and $20 \mathrm{~cm}$ from the tunnel walls in the case of PIV measurements. The smaller spanwise distance for PIV cases necessary to avoid impedance of laser optics due to existing tunnel hardware on the outer top of the wind tunnel. Additional attempts at producing the laser sheet from below the wind tunnel produced unfavorable refractive and reflective interference of the laser sheet.

\subsection{Hot-wire Anemometry}

Hot-wire anemometry data were collected using a Dantec StreamLine CTA (constant temperature anemometry) system and a Dantec Dynamics type 55P01 tungsten wire probe with Pt-W wire diameter of $5 \mu \mathrm{m}$, length of $3 \mathrm{~mm}$, and a sensing length of $1.25 \mathrm{~mm}$. In practice, the hot-wire is held to a constant temperature via voltage input. As passing wind flow cools the probe, voltage increases to maintain the required temperature and the voltage difference can be translated to velocity values for the passing flow. Acquisition of voltage data for all configurations took place over time periods of 300 seconds at $20 \mathrm{kHz}$ and 35 kHz. Velocities were calculated using King's law and Taylor's hypothesis. All measurements were taken at a downstream distance of $3 \mathrm{~m}$ from the tunnel spray grid, at vertical and minimum spanwise distances of $36.5 \mathrm{~cm}$ from the tunnel floor and wall, respectively. Measurements were conducted in an open-tunnel configuration (sans turbine) for calibration and to observe turbulence characteristics. To quantify wake behavior in the absence of particles, subsequent measurements were taken at the near and far wake locations as shown in Figure 3.1 ( $1 D$ and 9.6D, respectively). Power spectral density (PSD) of the measured instantaneous velocities were calculated for four carrier speeds $\left(U_{\infty}=2.61,4.86\right.$, and 8.3 $\mathrm{ms}^{-1}$ ) as shown in Figure 3.3. 


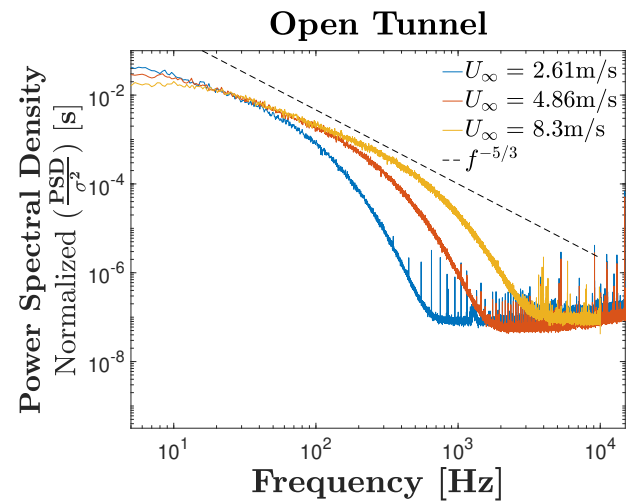

(A)

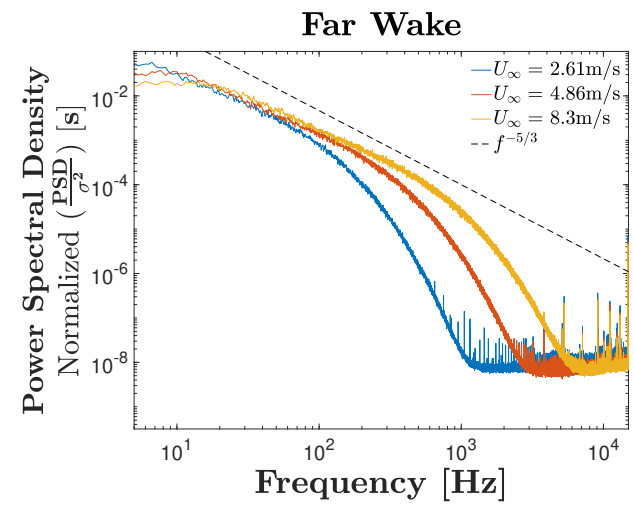

(C)

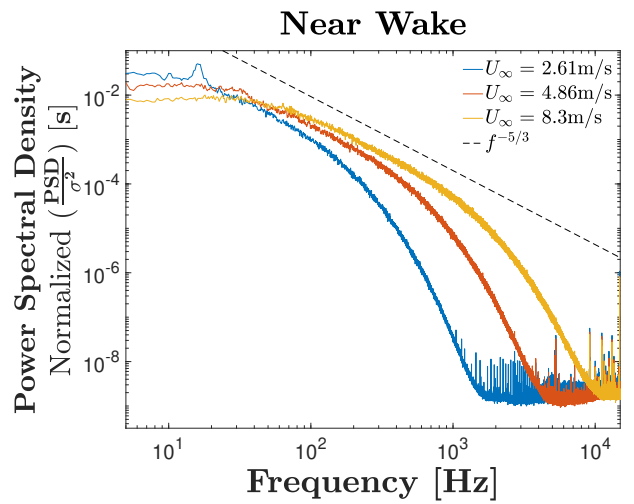

(B)

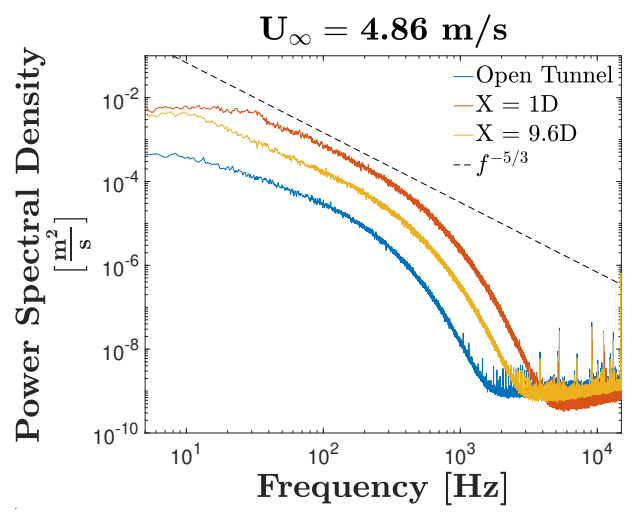

(D)

Figure 3.3: Power spectral density (PSD) from hot-wire measurements. Normalized PSD $\left(P S D / \sigma_{u}^{2}\right)$ at mean inflow velocities of $U_{\infty}=2.61,4.86$, and $8.3 \mathrm{~ms}^{-1}$ : (a) Open Tunnel, (b) Near wake of model $(1 D)$, and (c) Far wake of model (9.6D); (d) PSD for all three configurations at a single mean velocity $U_{\infty}=4.86 \mathrm{~ms}^{-1}$.

Figure 3.3(A) shows the PSD of the velocity signal $u$ normalized by the variance of instantaneous streamwise velocity $\left(P S D / \sigma_{u}^{2}\right)$ for the open-tunnel configuration. Figures 3.3(B) and 3.3(C) contain normalized PSD of data taken within the near and far wake of the rotor model, respectively. Figure 3.3(D) represents PSD for all three model configurations at a single inflow velocity of $U_{\infty}=4.86 \mathrm{~ms}^{-1}$. The variation in near wake energy content at frequencies $\leq 20 \mathrm{~Hz}$ and spike for the lower inflow velocity is likely due to slight offset of the hot-wire probe from tunnel center. The line with slope $f^{-5 / 3}$ represents Kolmogorov-based inertial range. Differences can be seen in terms of energy content between the open-tunnel and model cases, with the broadest range within the near wake. The near wake also more 
closely follows the $\mathrm{f}^{-5 / 3}$ slope than either the open tunnel or far wake cases. Also notable here is the mean streamwise velocity deficits which even in the far wake show $25 \%, 18 \%$, and $15 \%$ decreases for the $U_{\infty}=2.6,4.86$, and $8.3 \mathrm{~ms}^{-1}$, respectively. These differences point to lingering wake effects due to the presence of the rotor model even at a distance of 9.6D downstream. As seen in Figure 3.3(D), energy content increases within the model wake versus the open-tunnel configuration, with the largest difference represented by the near wake location. This emphasizes the effect of the rotor on turbulent energy within the flow.

\subsection{Phase Doppler Interferometry}

PDI measurements were taken within the near and far wake downstream of the turbine at the rotor hub height, $12 \mathrm{~cm}(1 D)$ and $115.2 \mathrm{~cm}(9.6 D)$, respectively, for each of the shaded cases in Table 3.1. PDI operates on detection of light refraction scattered due to particles passing through the intersection point of two laser beams of known frequency, resulting in a phase shift in the two beams observed by a receiving device. The difference in phase for each of the beams, along with the refractive properties of the particle, is then used to deduce particle diameter and velocity for each respective occurrence, assuming a spherical particle shape [32]. The PDI system used here was the PDI-200MD series from Artium Technologies, Inc., which uses two diode pumped solid state lasers, here green at a wavelength of 532 $\mathrm{nm}$ and blue at $491 \mathrm{~nm}$, each split into two beams of equal intensity. The green beams were vertically aligned and captured vertical velocity and particle size, while horizontally positioned blue beams captured streamwise velocity measurements. This system can detect particle diameters between 0.3 and $8000 \mu \mathrm{m}$ with a dimensional accuracy of $\pm 0.5 \mu \mathrm{m}$. The transmitter and receiver were positioned on opposing sides of the tunnel with respective focal lengths of $1000 \mathrm{~mm}$ and $500 \mathrm{~mm}$, fixated on the laser intersection point at hub height downstream of the turbine model. The PDI system remained stationary during all tests and 
the turbine was translated streamwise in reference to the intersection point for near and far wake locations. Receiver aperture was set to $200 \mu \mathrm{m}$. Signals induced from particle interactions within this intersection were then sent to the Artium advanced signal analyzer (ASA), which uses a Fourier-based Doppler burst detection algorithm to determine particle diameter and velocity for each instance. Maximum sampling frequency for the ASA is 320 $\mathrm{MHz}$, with a resolution of $0.01 \%$ of sampling frequency and 0.5 degree in phase. The resulting data were collected, organized, and exported within the proprietary Automated Instrument Management System (AIMS) version 5.2.

\begin{tabular}{|c|c|c|c|c|c|c|c|c|c|c|c|c|}
\hline $\mathrm{U}(\mathrm{m} / \mathrm{s})$ & \multicolumn{4}{|c|}{2.6} & \multicolumn{4}{|c|}{4.9} & \multicolumn{4}{|c|}{8.3} \\
\hline$R e_{D}$ & \multicolumn{4}{|c|}{$2.0 \times 10^{4}$} & \multicolumn{4}{|c|}{$3.8 \times 10^{4}$} & \multicolumn{4}{|c|}{$6.5 \times 10^{4}$} \\
\hline$R e_{\lambda}$ & \multicolumn{4}{|c|}{49.06} & \multicolumn{4}{|c|}{66.58} & \multicolumn{4}{|c|}{87.90} \\
\hline Flow Rate (L/min) & 0 & 1.2 & 1.7 & 2 & 0 & 1.2 & 1.7 & 2 & 0 & 1.2 & 1.7 & 2 \\
\hline$\Phi_{v}\left[x 10^{-5}\right]$ & NA & 0.77 & 1.1 & 1.3 & NA & 0.41 & 0.58 & 0.69 & NA & 0.24 & 0.34 & 0.4 \\
\hline
\end{tabular}

Table 3.1: Experimental cases: All cases represent PIV measurements, in both the near and far wake. Gray cells represent corresponding PDI measurements.

PDI data were obtained varying spray grid flow rates and inflow velocities for a minimum of 100,000 observations within the near and far wake of the turbine, where sampling frequency was dependent on particle crossing. Volume fractions $\left(\Phi_{\nu}\right)$ range from $\sim 9.7 \times 10^{-6}$ to $2.58 \times 10^{-5}$. Figure 3.4 (A) represents the probability density function (PDF) of particle diameter for three inflow velocities. The top two subfigures represent measurements within the far wake of the turbine, and bottom two show near wake. The two left subfigures represent a uniform injection rate of $1.7 \mathrm{~L} / \mathrm{min}$ and the right at $2 \mathrm{~L} / \mathrm{min}$ from the spray grid. In this figure, dotted lines denote measurements taken in the model wake. Solid lines represent measurements taken with no model present (open-tunnel) immediately prior to their wake counterparts. The bar plot in Figure 3.4(B) expresses mean diameter for a single inflow rate of $R e_{\lambda}=66.58$. Shaded bars indicate results within the turbine wake, white bars represent open-tunnel, bars with and without slanted stripes are at injection rates of 1.7 $\mathrm{L} / \mathrm{min}\left(\Phi_{1.7}\right)$ and $2 \mathrm{~L} / \mathrm{min}\left(\Phi_{2}\right)$ respectively, and bars with and without horizontal stripes represent near and far wake locations. 

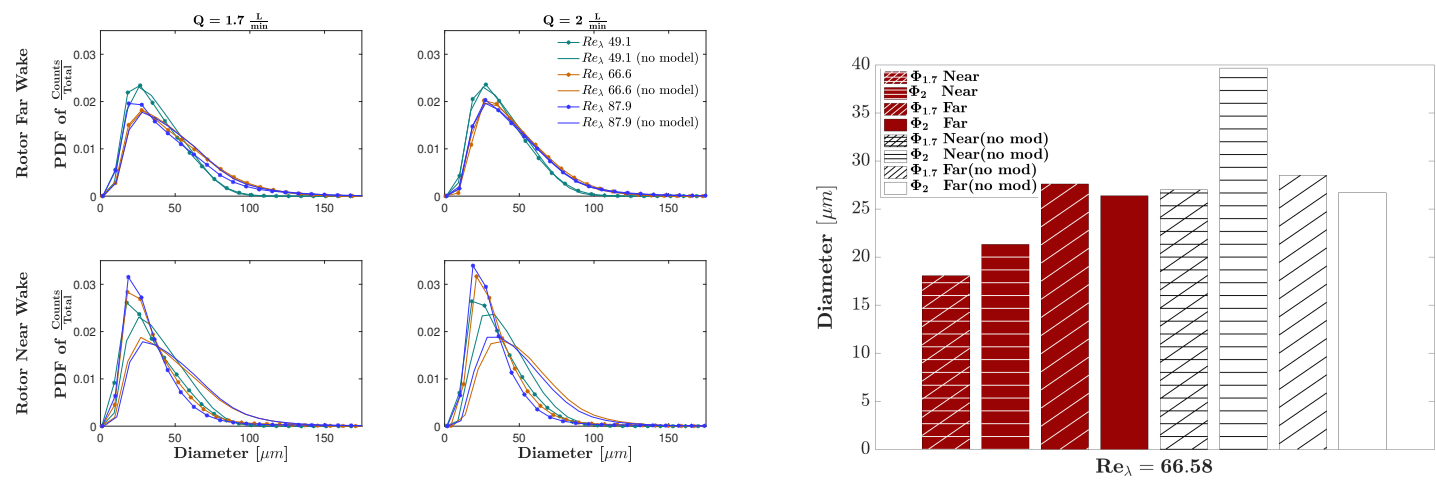

(A)

(B)

Figure 3.4: PDI-captured particle diameters: (a) PDF of diameter counts. Solid lines represent open-tunnel (i.e., sans turbine). Top two subfigures show far wake $(9.6 D)$ for water injection flow rates 1.7 and $2 \mathrm{~L} / \mathrm{min}$ (left and right). Lower plots show near wake (1D); (b) Mean diameter bar plot for varied injection rates, wake location, and model presence. Slanted stripes denote injection of $1.7 \mathrm{~L} / \mathrm{min}$, horizontal are near wake locations, colored bars represent model present, and white bars open-tunnel.

Measurements within the far wake show similarity in diameter values with regard to open-tunnel versus model presence as seen in Figures 3.4(A) and 3.4(B). In the near wake however, smaller diameters are highly prevalent versus the open-tunnel configuration. This suggests smaller particles are being captured within the turbine wake and points to particle entrainment within the wake region. As discussed in Section 2, particles with small diameter, and ultimately small relaxation times $\left(\tau_{p}\right)$ tend to be found within vortical structures as they are entrained into the large-scale structures of turbulent wakes. The presence of smaller particles within the near wake region then highlights this behavior and speaks to energy gained within the wake from ambient flow.

\subsection{Particle Image Velocimetry}

Particle Image Velocimetry (PIV) is a form of image analysis, where relatively high speed photos are taken of particles within a flow field. The particles often used are droplets of low-density, buoyant vapor known as seeding. A thin, planar focused laser sheet is emitted within the interrogation space illuminating only a select area or volume of particles to be 
captured as images. The snapshots are then compared in sequence to identify trajectories of particle groups from frame to frame. Velocities are calculated based on known spatial attributes and time difference between successive images. For this study, PIV data were taken for all cases shown in Table 3.1. The laser sheet was produced using a diode-pumped Nd:YLF laser (Litron LD30-527), emitting a laser with wavelength of $527 \mathrm{~nm}$, directed and focused downward from the top of the tunnel. The laser sheet was centered spanwise with respect to the turbine, producing a velocity field in the streamwise and vertical plane $(x-y)$. Images of the laser sheet were captured using a Phantom V2511 with a full resolution of $1280 \times 800$ megapixels. Interrogation areas for all cases were vertically centered about the turbine hub height with a total plane vertical dimension of $25 \mathrm{~cm}( \pm 1.04 D)$. Streamwise PIV plane dimensions were a minimum of $11.5 \mathrm{~cm}(0.8 D$ to $1.7 D)$ and $18.8 \mathrm{~cm}(9.4 D$ to $11 D$ ) for near and far wake, respectively. Variations in captured image spatial dimensions, especially for the near wake, were due to avoidance of adverse reflections from the turbine model. Images from the Phantom camera were sent to, and processed with Dantec Dynamics proprietary software and the open source PIVlab platform built for MATLAB. For inertial particle cases, water droplets from the spray grid were treated as inertial seeding for PIV data capture and all quantities are based solely on particle behavior. To compare single-phase flow behavior with that of the polydisperse flow cases, smoke machine vapor was used for PIV measurements with sub-inertial particles. A minimum of 2000 snapshots were taken for each of the shown cases. For the $5 \mathrm{~Hz}$ tunnel speed and a water flow rate of $2 \mathrm{~L} / \mathrm{min}$, approximately 10000 snapshots were taken to test convergence, finding that the number of snapshots needed for statistical convergence was below 2000. 


\section{Chapter 4}

\section{Results}

\subsection{Phase Doppler Interferometry}

Initial observations of effects on streamwise velocity while varying $R e_{\lambda}$ and injection rate $\Phi_{v}$ were conducted by PDI and shown in Figure 4.1, where probability density functions (PDFs) with respect to PDI velocity observations at hub height are shown for near and far wake locations downstream the turbine model $(1 D$ and 9.6D). Two injection rates corresponding to 1.7 and $2 \mathrm{~L} / \mathrm{min}\left(\Phi_{\nu}=\Phi_{1.7}, \Phi_{2}=0.58,0.69\right)$ were imposed at a range of three inflow velocities $\left(R e_{\lambda}=49.06,66.58,87.9\right)$. The left and right columns are increasing water injection rates from the grid, while top and bottom rows are the far and near wake respectively. Dotted lines represent measurements taken within the model turbine wake. Solid lines represent data taken with no model present in the tunnel immediately prior to each respective model run. Velocity deficits are visible in both the near and far wake versus the open-tunnel configuration, pointing to wake continuance even at 9.6D. Values of these deficits vary with higher $R e_{\lambda}$ cases and higher injection rates $\left(\Phi_{v}\right)$. The lower $\Phi_{v}$ cases $\left(\Phi_{\nu}=\Phi_{1.7}\right)$ show deficits ranging from 1.37 to $2.7 \mathrm{~ms}^{-1}$ in near wake and 0.29 to $0.74 \mathrm{~ms}^{-1}$ in far wake, while with increased $\Phi_{v}\left(\Phi_{v}=\Phi_{2}\right)$, deficits range from 1.44-2.84 $\mathrm{ms}^{-1}$ and $0.33-1.00 \mathrm{~ms}^{-1}$ in near and far wake, respectively. The large sensitivity to $\Phi_{v}$ within the far wake suggests that particle concentration has an exacerbating effect on wake persistence and intensity. 

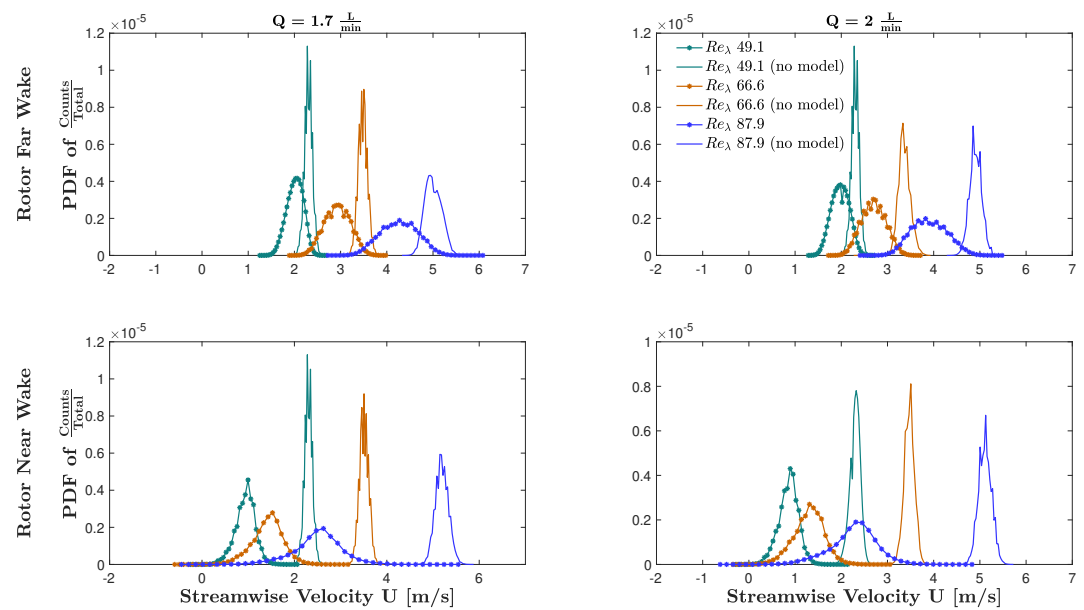

Figure 4.1: PDF of count ratio for instantaneous streamwise velocity within the turbine wake. Solid lines represent velocity distributions of open-tunnel configuration (no turbine present). Top row subfigures, left and right, represent data collection at the far wake location $(9.6 D)$ for a uniform distribution of water injected at flow rates 1.7 and $2 \mathrm{~L} / \mathrm{min}$ respectively from the spray grid. Bottom row subfigures represent data collected within the rotor near wake $(1 D)$ at each of the respective flow rates

Isolating effects of inertial particle imposition for a single inflow velocity, comparison of PDF versus velocity is plotted from PDI data for $R e_{\lambda}=66.58$ in Figure 4.2. Triangle markers represent measurements within the near wake region at $1 D$, circle markers represent far wake at 9.6D downstream of the model, and solid lines represent measurements within the open-tunnel configuration prior to the corresponding injection and location measurement. At this inflow velocity, respective deficits for $\Phi_{1.7}$ in near and far wake are $59.5 \%$ and $15.83 \%$ and $\Phi_{2}$ deficits are $62.61 \%$ and $19.83 \%$. 


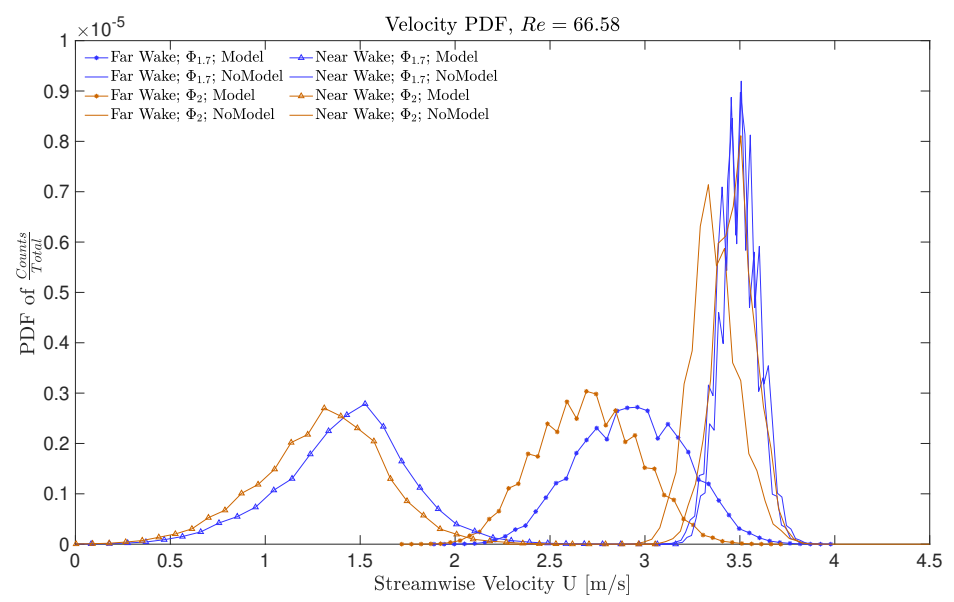

Figure 4.2: PDF of count ratio for instantaneous streamwise velocity within the turbine wake for $R e_{\lambda}=66.58$. Solid lines represent velocity distributions of open-tunnel configuration (no turbine present).

While deficits within the turbine near wake regions are expected to be higher than those in the far wake, the notable velocity decreases in the far wake region highlight incomplete wake recovery and suggest that energy content is still affected at $9.6 \mathrm{D}$ downstream. With reference to observations in Figure 3.4(B) and in terms of entrainment, the larger particle diameters present in the far wake are more likely to be following the saddle regions of large structures rather than entrained within vortical regions. Between the two injection rates, $\Phi_{2}$ shows higher velocity deficits than $\Phi_{1.7}$ on the order of 3-4\%, suggesting that with increased volume fraction (ie. heavier rains or thicker debris fields) the behavior within the turbine wake is affected and wake effects are prolonged.

\subsection{Particle Image Velocimetry}

Planar images of streamwise velocity deficits $\left(U_{\infty}-U\right) / U_{\infty}$ within the turbine model wake were obtained from PIV data and shown in Figure 4.3. Each column set $\left(R e_{\lambda}=49.06\right.$, 66.58, and 87.90) contains two planes depicting near and far wake $(0.75 D-1.75 D$ and 9.45D-11D). The four rows represent increasing water injection volume fractions from top to bottom, where $\Phi_{0}$ represents sub-inertial seeding (tracers), and $\Phi_{1.2}, \Phi_{1.7}$, and $\Phi_{2}$ 
correspond to water injection rates from a grid of $1.2,1.7$ and $2 \mathrm{~L} / \mathrm{min}$. Volume fractions for all injection rates at each $R e_{\lambda}$ are shown in Table 3.1. The $\Phi_{0}$ cases were obtained with smoke as sub-inertial tracers, and act as a baseline to show wake behavior in the absence of inertial particles. As noted in Section 3 PIV data for the inertial cases $\left(\Phi_{1.2}, \Phi_{1.7}\right.$, and $\Phi_{2}$ ) were captured by treating the particles as inertial 'seeding' and filtering was employed to minimize the effect of light refraction.
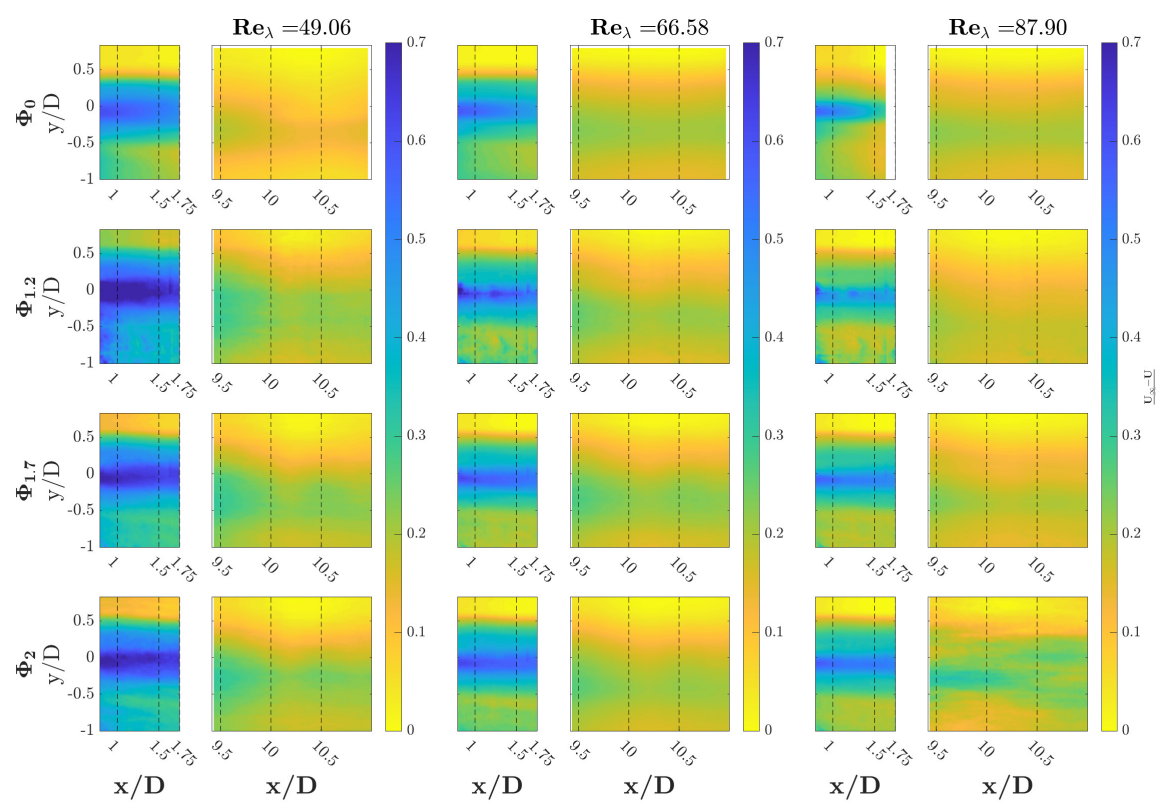

Figure 4.3: Planar wake contours of normalized streamwise velocity deficits $\left(U_{\infty}-U\right) / U_{\infty}$ for three values of Taylor-based Reynolds number $\left(R e_{\lambda}\right)$ increasing from left to right, and four volume fractions $\left(\Phi_{v}\right)$ increasing in rows from top to bottom.

Figure 4.3 emphasizes changes in wake width and velocity deficits with respect to inertial particle presence. Near wake for all injection rates produces diminishing wake widths and deficits with increase in $R e_{\lambda}$, agreeing with wake behavior of the sub-inertial case and with literature [20]. In the near wake, wake width and deficit magnitudes increase with $\Phi_{v}$ for all $R e_{\lambda}$ with the exception of $R e_{\lambda}=49.06$. Unlike the higher $R e_{\lambda}$ cases, where the wake regions diverge with elevated injection rates, the lower inflow velocity shows little effect due to increased particle presence once the droplets are present. All polydisperse cases at this inflow, $\Phi_{1.2^{2}}-\Phi_{2}$, exhibit notable similarity in wake width and velocity deficits, differ- 
ing from the $\Phi_{0}$ case only through decreased velocity magnitude. The effects of $\Phi_{v}$ on the wake are less apparent between the multi-phase cases $\Phi_{1.2}-\Phi_{2}$, yet the multi-phase flow cases show expanded and less-central wakes compared to the single-phase flow cases. This would suggest that at lower carrier velocities, the near wake is less affected by the imposition of multiphase flow than at higher $R e_{\lambda}$ inflow conditions. This behavior agrees with the formerly discussed PDI results and the sphere wake results of Homann and Bec, where increased turbulence at higher inflow velocities has a greater influence particle behavior and concentration within wake flow [22].

In the far wake, presence of inertial particles intensifies lower-velocity regions within the wake center. The wake behavior in this region differs between all three inflow velocities. For $R e_{\lambda}=49.06$, the wake width increases between $\Phi_{0}$ and $\Phi_{1.2}$ where a wide, low-velocity region appears upstream of $x=1 D$ expanding to nearly to the bottom on the rotor profile. As $\Phi_{v}$ increases, the wake width diminishes ultimately producing a more persistent lowvelocity wake center at $\Phi_{2}$.

The moderate inflow velocity case of $R e_{\lambda}=66.58$ exhibits a horizontal wake constancy with single-phase flow, but the shape of the wake is disrupted by the influence of particles, where the low velocity region for $\Phi_{1.2}$ is broken between $10 D-10.5 D$ with broader low velocity regions on either side. At the injection rate of $\Phi_{1.7}$, the wake is at its widest with a large deficit region throughout the plane. At the highest injection rate, the wake appears to contract and the low velocity region is again focused at the center of the wake.

The highest inflow velocity at $R e_{\lambda}=87.9$ demonstrates the greatest dependence on multi-phase particle injection. Within the single-phase far wake, a persistent wake is visible with a defined, low-velocity center region. As $\Phi_{v}$ increases, the wake width expands and lowest velocity region intensifies beyond the lower turbine profile. Significantly for the $\Phi_{2}$ case, the entire wake profile as widened beyond the plane of view. There also appears to be a low-speed core, about which streaks of lower velocity are induced. The broadened and continuous wake region demonstrates a more complicated and prolonged recovery within 
high speed, high concentration wakes than that of single-phase.

The remainder of this discussion focuses on the moderate inflow velocity case, $R e_{\lambda}$ $=66.58$, due to behavior variation between $\Phi_{v}$ values as discussed above and to isolate effects of particle presence. Statistical quantities for the near wake within this carrier flow are presented as PIV contours in Figure 4.4, with columns from left to right representing mean streamwise velocity $U$, mean vertical velocity $V$, streamwise Reynolds normal stress $\overline{u^{\prime} u^{\prime}}$, Reynolds shear stress $\overline{u^{\prime} v^{\prime}}$, and vertical Reynolds normal stress $\overline{v^{\prime} v^{\prime}}$. All statistics are normalized by the local freestream velocity $U_{\infty}$ with respect to quantity units.
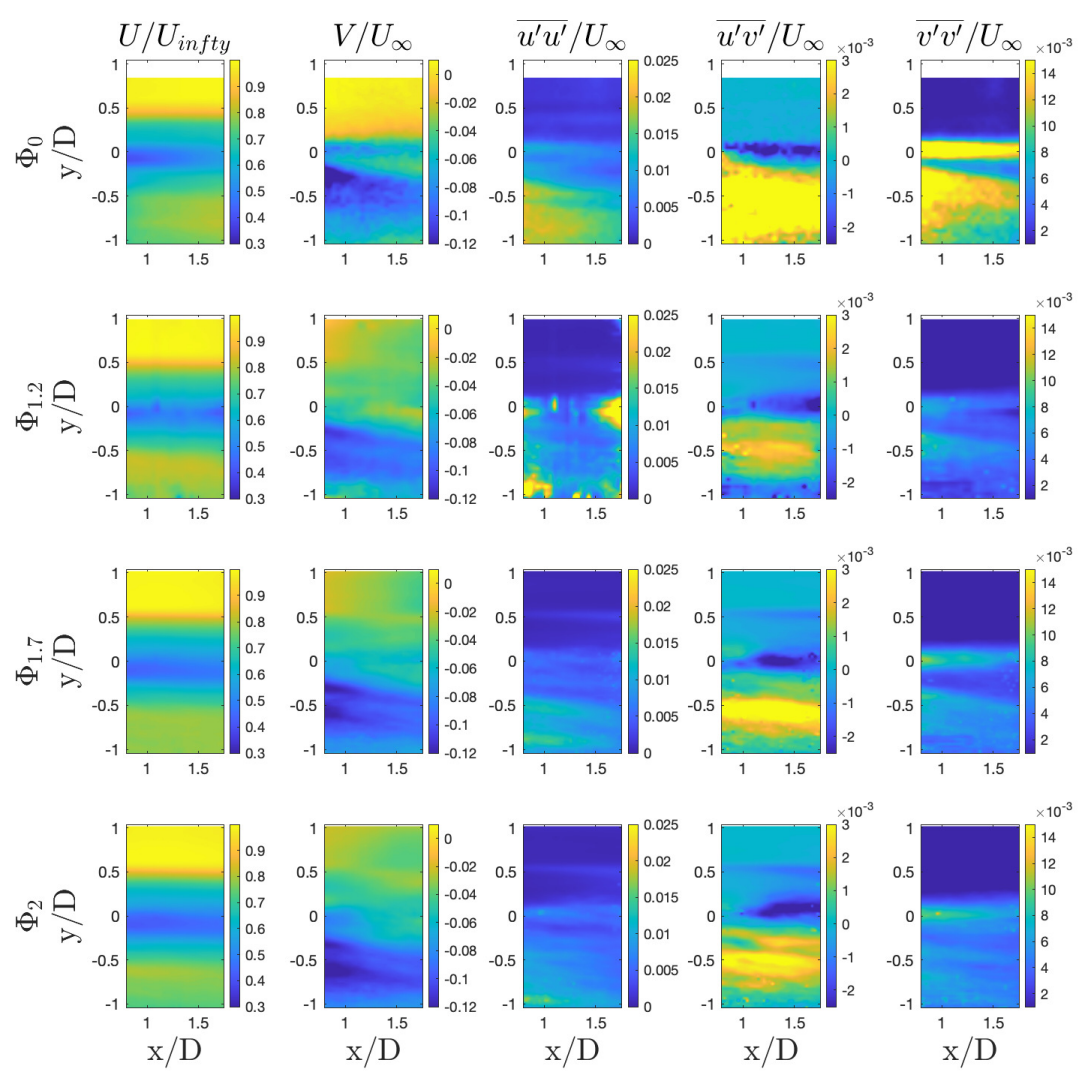

Figure 4.4: Near wake, normalized mean statistics. Left to right: Streamwise velocity $U / U_{\infty}$; Vertical velocity $V / U_{\infty}$; Reynolds Stresses $\overline{u^{\prime} u^{\prime}} / U_{\infty}^{2} ; \overline{u^{\prime} v^{\prime}} / U_{\infty}^{2} ; \overline{v^{\prime} v^{\prime}} / U_{\infty}^{2}$

The effects of near wake statistics are first discussed in relation to Figure 4.4. Streamwise velocity $U / U_{\infty}$ for $\Phi_{0}$ represents the sub-inertial case and shows a defined wake core 
at hub height, diminished velocity within the rotor profile, and with a large velocity gradient beginning just below the rotor top to freestream velocity at $y / D \sim 0.5$. Below the rotor, $U / U_{\infty}$ is diminished due to the tower, increasing slightly in the downstream portion of the interrogation window. With the introduction of inertial particles at $\Phi_{1.2}$, the wake core is intensified by lower streamwise velocities. As $\Phi_{v}$ increases, hub height velocity values are qualitatively similar, yet the width and extent of this deficit also increase producing a near constant wake core across the plane for $\Phi_{2}$. The rotor shadow is expanded beyond $y / D=0.5$ between $\Phi_{0}$ and $\Phi_{1.2}$, and the gradient of the wake to freestream velocity at the rotor top is contained within a smaller region. This remains constant for all inertial cases $\Phi_{1.2}-\Phi_{2}$. Additionally, particle presence enhances streamwise velocities below the rotor, producing regions of increased $U / U_{\infty}$ beginning at $y / D \sim-0.6$ across the entire downstream plane compared to the stalled motion in $\Phi_{0}$.

Vertical velocity $(V)$ is also affected by injection rate increases. $\Phi_{0}$ contains a streak of downward motion at hub height and a more intensified falling region at $y / D \sim-0.2$ to -0.6 close to the rotor, with near $V / U_{\infty}=0$ values above hub height. At injection concentration of $\Phi_{1.2}$, the effects within hub height diminish along with the intensity of downward motion within the lower half of the rotor, but the regions containing falling particles broaden across the plane. At this injection rate, regions of downward motion below hub height intensify across the $x / D$ direction at $y / D \sim-0.1$ extending to $y / D \sim-1$, highlighting the downward propulsion of the particles induced by hitting the rotor and tower. Increases in $\Phi_{v}$ result in return of effects at hub height and produce significant increases in downward motion below hub height. At $x / D=0.75, \Phi_{1.7}$ and $\Phi_{2}$ a shadow of decreasing vertical velocity just below hub height begins to appear $(y / D \sim-0.2)$ and effects at the rotor bottom edge are intensified $(y / D \sim 0.5)$, sloping downward and extending to $x / D=1.5$. Effects of increased concentration on upward motion above hub height shows minimal change between injection rates compared to the intensified downward propulsion induced below the rotor hub. This contrast within the near wake highlights a greater tendency of particles to fall in response 
to direct impact with the rotor blades.

The normal Reynolds stress term $\overline{u^{\prime} u^{\prime}} / U_{\infty}^{2}$ is presented in the central column of Figure 4.4 and represents the contributions of streamwise fluctuations to turbulent kinetic energy within the wake. Within the rotor shadow of the sub-inertial case $\Phi_{0}$, a slight band of increased energy is visible at $y / D \sim 0.3$, and a band of higher energy can be seen at hub height with similar magnitudes at $y / D=-0.25$. Along the rotor bottom, $\overline{u^{\prime} u^{\prime}} / U_{\infty}$ increases and a local maxima is visible in the tower wake. With the inclusion of inertial particles at $\Phi_{1.7}$, these high energy regions mimic $\Phi_{0}$ in shape, but a band appears above the rotor top at $y / D \sim 0.6$, the width at hub height increases, and the lower rotor region becomes continuous downward from $y / D=-0.25$. Magnitudes at and below hub height locations are significantly pronounced in $x / D$ locations nearest the rotor model and begin to separate into two concentrated regions near $x / D=1.75$. Magnitudes within these regions increase with particle concentration, where $\Phi_{2}$ represents the highest values for all cases at $y / D \sim$ 0.6 , nearest the rotor for $y / D \leq-0.4$, and three high energy streaks appear beginning at $x / D=1$.

The shearing Reynolds stress term $\overline{u^{\prime} v^{\prime}} / U_{\infty}^{2}$ in the fourth column highlights the influence of vertical fluctuations on streamwise momentum flux. In the sub-inertial case $\Phi_{0}$, significant positive shearing is produced by the rotor bottom edge and negative at hub height. The sloping profiles emphasize the effect of falling particles on the near wake momentum. Along the top half of the rotor, slight negative quantities are present, yet the effects are less concentrated and are much lower in magnitude than the hub height and bottom rotor regions. In contrast to the sub-inertial case, the onset of inertial particles causes shearing regions to expand and lessen in magnitude, while introducing a new region along the rotor edge. At $\Phi_{1.2}$, the hub height band becomes separated in the streamwise direction, and shearing magnitudes in the top half of the rotor are more visible. A concentrated high content band is still visible at $y / D \sim-0.5$, but is not constant over the whole streamwise domain and is surrounded by moderate levels in and beyond the bottom half of the rotor pro- 
file which is bounded by a region of negative magnitudes approaching $y / D \sim-0.75$. With the increase in particle concentration, magnitude concentration at hub height and rotor bottom becomes more constant streamwise across the domain, reminiscent of the sub-inertial case, but the magnitudes are still much diminished. At $\Phi_{2}$, the top half of the rotor shadow contains greater variability than in the subinertial case, but is still minimal compared to the shearing present at the bottom rotor edge. Previous studies have shown this quantity to be equal in absolute value magnitude between top and bottom rotor edges for turbine wakes, suggesting that tower effects in this study may be more pronounced [20]. However, the noticeable band of shearing developed between the top of the rotor and throughout the wake shadow at $\Phi_{2}$ suggests turbulence enhancement due to particle presence.

The last column in Figure 4.4 contains the normal Reynolds stress $\overline{v^{\prime} v^{\prime}} / U_{\infty}$ which speaks to energy contributions within the wake from the vertical fluctuations. In the absence of particles, $\Phi_{0}$ mimics the profile seen for vertical velocity $V / U_{\infty}$, but highlights maximum energy contributions at hub height persisting to $x / D \sim 1.5$. Nearest to the turbine, the bottom rotor half shows significant magnitudes which follow in a sloping pattern downstream. This effect diminished slightly below the rotor bottom, yet still emphasizes contributions due to tower presence compared to the region above hub height. The introduction of particles again minimizes the concentration of energy due to this term, by lessening magnitude in the hub height and lower regions while expanding the affected region across $y / D$. While the $\Phi_{2}$ hub height band magnitude is diminished, the region appears to be expanding in width downstream of the model, and below $y / D \sim-0.25$ the effects of impact with the rotor and tower are visible throughout the $x / D$ domain. The expanded profile imposed in the lower half of the domain emphasizes the effect of particle impact with the turbine on wake energy content and suggests subsequent effects on particle settling within the near wake.

Figure 4.5 contains normalized mean statistics within the far wake of the turbine model $(\sim 9.5 D$ to $11 D)$. The rows and columns are organized in the same manner as Figure 4.4, 
where rows represent increasing particle concentration from top to bottom, and the columns from left to right contain the quantities $U / U_{\infty}, V / U_{\infty}, \overline{u^{\prime} u^{\prime}} / U_{\infty}^{2}, \overline{u^{\prime} v^{\prime}} / U_{\infty}^{2}$, and $\overline{v^{\prime} v^{\prime}} / U_{\infty}^{2}$, respectively.
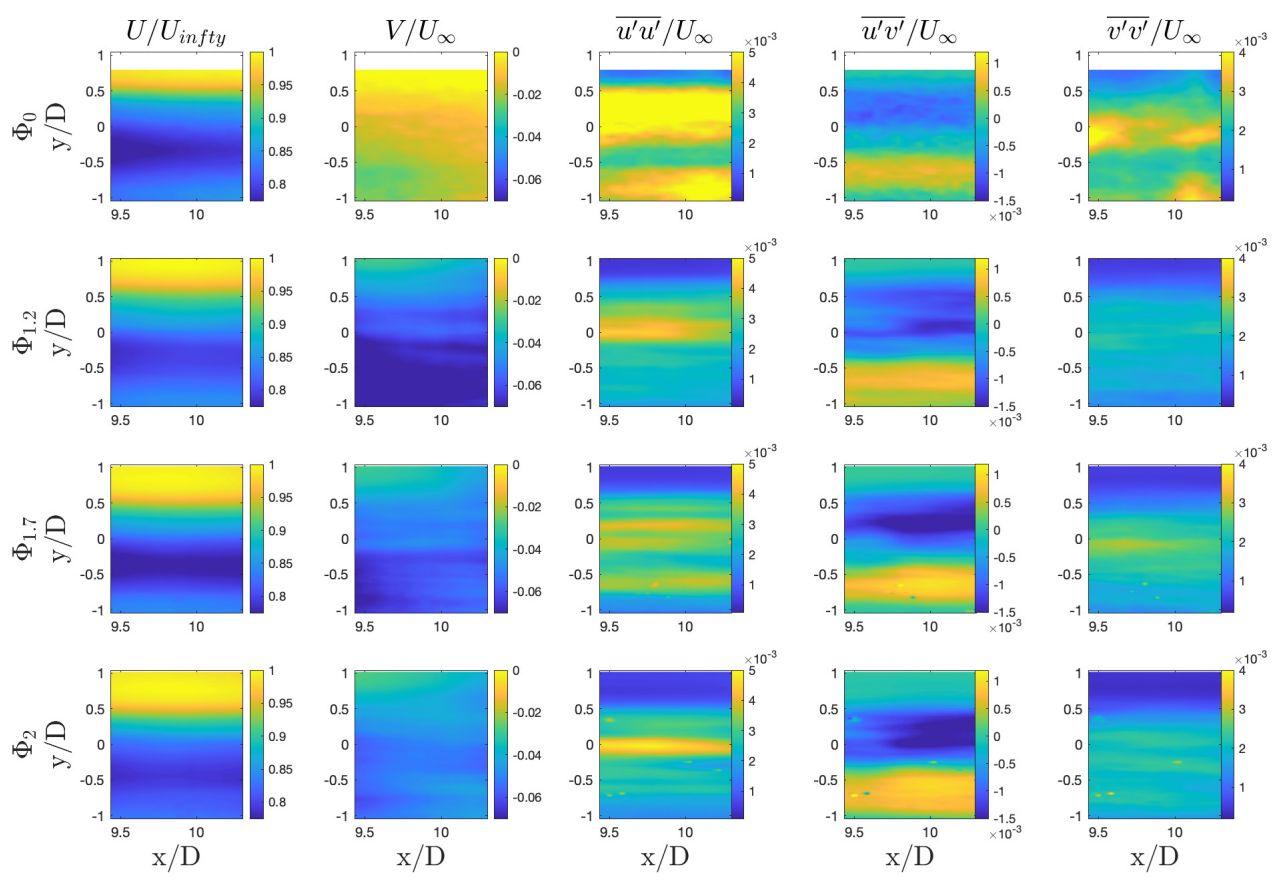

Figure 4.5: Far wake, normalized mean statistics. Left to right: Streamwise velocity $U / U_{\infty}$; Vertical velocity $V / U_{\infty}$; Reynolds Stresses $\overline{u^{\prime} u^{\prime}} / U_{\infty}^{2} ; \overline{u^{\prime} v^{\prime}} / U_{\infty}^{2} ; \overline{v^{\prime} v^{\prime}} / U_{\infty}^{2}$.

Streamwise velocity $U / U_{\infty}$ demonstrates continued propagation within the far wake, where the wake core is centered about the region of $y / D \sim-0.3$ for all $\Phi_{\nu}$ cases. The wake in $\Phi_{0}$ is bounded at the top of the rotor by a sharp gradient to freestream velocity and drops below the plane of view. The primary deficit region is situated on the bottom half of the rotor shadow, decreasing in width over the streamwise plane. With the introduction of water droplets at $\Phi_{1.2}$, velocity within the core increases slightly, but its width is more consistent as it projects downstream than the smoke case. The upper portion of the wake is expanded beyond the upper rotor edge and the gradient region thickens, suggesting increased particleflow interactions outside the rotor edge. As $\Phi_{v}$ increases, the wake is again bounded at at $y / D=0.5$, but for $\Phi_{1}$ the concentrated wake region slows and begins to expand below the 
rotor profile. For the highest inject rate, $\Phi_{2}$, the lowest velocity is maintained along the rotor bottom. Deficit magnitudes are lessened overall for this case compared to $\Phi_{0}$ through $\Phi_{1.7}$, and wake effects are more evenly distributed in the lower half of the plane. For all concentration levels, wake features in this far downstream region correlate with the turbine model geometry which highlight rotor wake persistence even beyond the plane of view. The entire far wake rotor profile shadow is subject to $10-25 \%$ decreases from freestream velocity which could significantly diminish the efficiency of a subsequent turbine if present.

Differences between the sub-inertial and particle-laden cases are more prominent in the vertical velocity contours of column 2 . In the $\Phi_{0}$ case, the rotor wake appears below $y / D$ $=0.5$, with slight effects of the tower visible below the rotor profile at $y / D=-0.5$. At the lowest injection rate, the propensity of falling particles is apparent over the entire visible plane, with increases in downward trajectory visible at the rotor top and most affected below hub height. As more particles are introduced into the flow, velocity magnitudes appear to regain consistency throughout the contour region but particles within the space below hub height are still pushed downward marking lingering effects from the upstream wake.

Column 3 of Figure 4.5 again speaks to kinetic energy contributions due to streamwise velocity fluctuations. For $\Phi_{0}, \overline{u^{\prime} u^{\prime}} / U_{\infty}$ dominates in the upper half of the rotor and below the rotor profile, separated by a lower magnitude region on the rotor bottom half. The disruption in this lower region is a relic of the rotor and mimics the wake core position seen in the first column for $U / U_{\infty}$. Once particles are injected, the overall magnitude of $\overline{u^{\prime} u^{\prime}} / U_{\infty}^{2}$ diminishes greatly, but a band of considerable magnitude appears surrounding hub height for $\Phi_{1.2}$. The moderate injection rate $\Phi_{1.7}$ separates into three distinct regions of energy injection, two symmetrical streaks around hub height, and one below the rotor profile at $y / D \sim-0.75$. At the highest injection rate, the hub region again concentrates into a single band of increased magnitude. The differences between the sub-inertial and particle-laden cases here suggest that the dense particles are less sensitive to streamwise fluctuations within the flow than the buoyant seeding. 
The shearing Reynolds stress term $\left(\overline{u^{\prime} v^{\prime}} / U_{\infty}^{2}\right)$ in this location, representing momentum flux due to turbulence, further mimics the symmetrical behavior of turbine wakes, suggesting diminished tower effects within the far wake region. For the sub-inertial case, the primary regions are aligned with around the wake core visible in the Streamwise velocity. At $\Phi_{1.2}$, both regions expand in size, with high absolute magnitude regions nearly encompassing the entire wake shadow and increasing in intensity. This aligns with the increased downward motion of dense particles within a flow field. Increasing $\Phi_{v}$ further sharpens the contrast of shearing effects and momentum contributions due to particle presence. The maximum injection rate $\Phi_{2}$ returns to the symmetry of separated high intensity regions about the corresponding wake core of $U / U_{\infty}$. Magnitudes are greatest at this particle concentration and appear to increase with streamwise distance, pointing to increased turbulent energy within the wake even beyond the visible plane.

Vertical fluctuation contributions to momentum displayed as $\overline{v^{\prime} v^{\prime}} / U_{\infty}$ again show significant differences between the sub-inertial and particle-laden cases. With the single-phase smoke case, high energy content is visible at hub height throughout the far wake. With particles present in $\Phi_{1.2}$, the energy content diminishes in magnitude and is relatively continuous throughout the rotor shadow. Increasing $\Phi_{v}$ to $\Phi_{1.7}$ introduces streaking regions centered about hub height mimicking the vertical velocity behavior for the same case. Flux contributions at $\Phi_{2}$ are again strengthened and redistributed into three defined streaks, two symmetrically located around hub height and one at $y / D \sim-0.75$. The presence of particles thus lowers the contributions of vertical momentum compared to a single phase flow, but this effect diminishes with increased particle concentration. This effect may be due to the slight decrease in particle size observed for higher injection rates within the far wake in Figure 3.4 and possible increases in gravitational effects. Temporally and spatially averaged flow statistics at $R e_{\lambda}=66.58$ are represented as vertical profiles for all particle injection rates in Figure 4.6. Spatial averaging here considers a total of $2 \mathrm{~mm}$ streamwise, centered about near and far wake locations $(1 D$ and $9.6 D$ respectively). For all profiles, solid and 
dashed lines represent near and far wake values, respectively. Streamwise velocity $U$ for all particle concentrations follow similar profile shape, but the changes in near wake width from Figures 4.3 and 4.4 are again apparent. In the far wake, $U / U_{\infty}$ is nearly collapsed below hub height, yet beginning in the upper rotor region, $U / U_{\infty}$ profiles for $\Phi_{1.7}$ and $\Phi_{2}$ show greater deficit than $\Phi_{0}$. Nearing the top of the rotor, $\Phi_{1.2}$ diminishes compared to $\Phi_{0}$ representing the extended wake width observed in the contours of Figure 4.5.
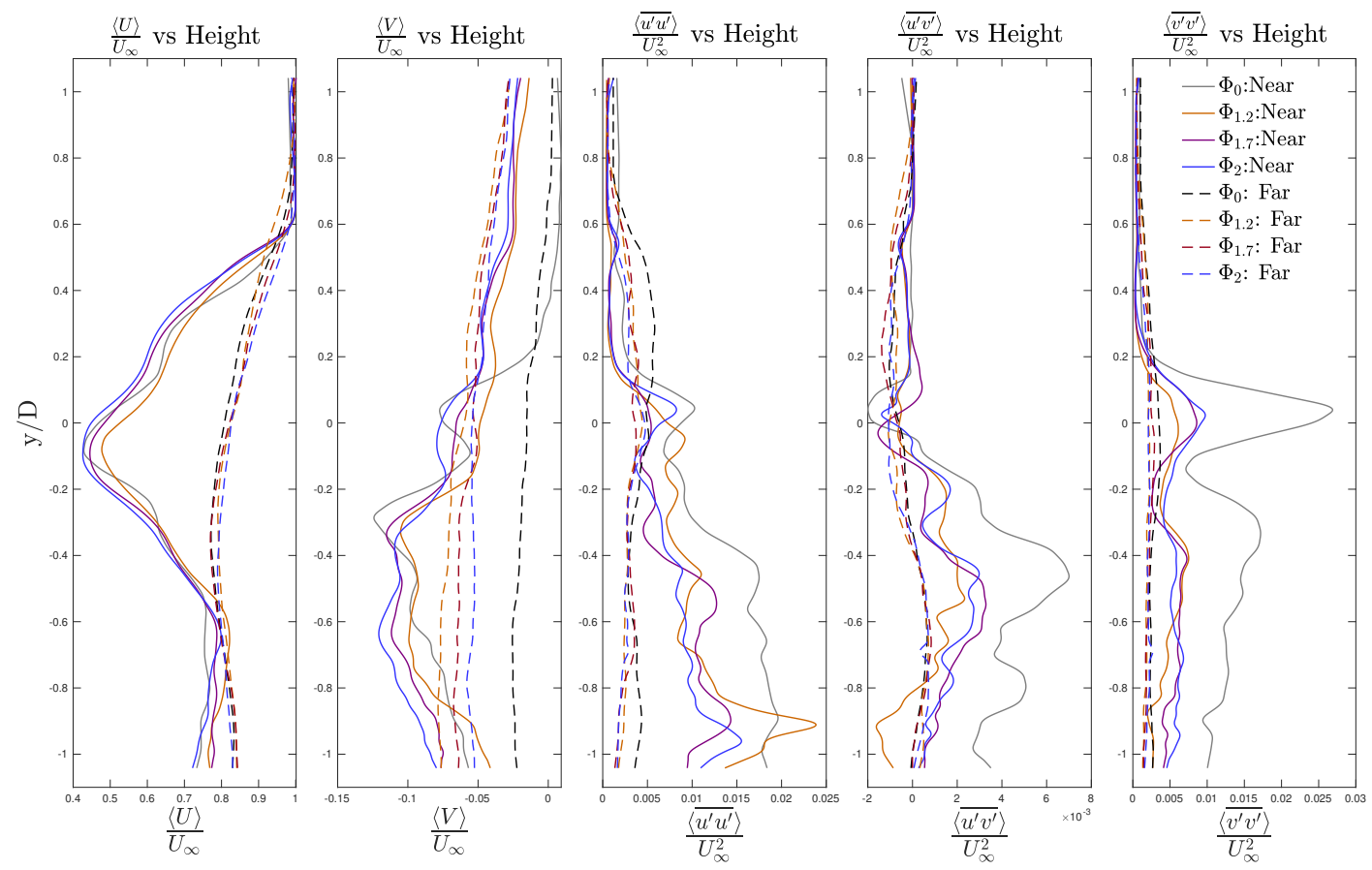

Figure 4.6: Vertical profiles of normalized, time-averaged statistics, spatially averaged over 1 $\mathrm{cm}$ within near and far wake of the turbine model $(1 D$ and 9.6D). Dotted lines represent far wake locations. Left to right: Mean streamwise velocity (U); vertical velocity (V); and standard deviation $\left(\sigma_{u}\right)$; Reynolds stresses $\left.\left\langle\overline{u^{\prime} u^{\prime}}\right\rangle ; \overline{\left\langle u^{\prime} v^{\prime}\right.}\right\rangle ;\left\langle\overline{v^{\prime} v^{\prime}}\right\rangle$

Vertical velocity in the near wake emphasizes increased downward motion for the higher concentration cases $\Phi_{1.7}$ and $\Phi_{2}$ versus sub-inertial and $\Phi_{1.2}$ cases at the bottom of the rotor profile and above $y / D=0.1$. Differences in behavior are less distinct within the bottom half of the rotor shadow, where near wake vertical velocities of the sub-inertial case peaks between $y / D \sim-0.2$ to -0.4 and just above hub height. Above which, the $\Phi_{0}$ case quickly returns to that of the freestream region, while inertial particle cases collapse 
into a falling trajectory. Within the far wake, the downward motion of falling particles is further emphasized vs the sub-inertial case over the entire vertical section and increased particle concentration produces increases in $V / U_{\infty}$ over the rotor profiles.

The streamwise contributions to kinetic energy $\left(\overline{u^{\prime} u^{\prime}} / U_{\infty}\right)$ in the near wake are most affected by particle presence within the bottom half of the rotor, where the $\Phi_{1.7}$ and $\Phi_{2}$ cases show lower values versus $\Phi_{0} . \Phi_{1.2}$ exceeds all cases except at $y / D \sim-0.1$ and $y / D \sim$ -0.9 . Beginning in the top half of the rotor, all particle cases collapse to values still less than that of $\Phi_{0}$, continuing up into the freestream region. In the far wake, streamwise energy contributions are similar for all inertial particle cases, remaining less than that for $\Phi_{0}$ throughout the profile. The lower values for higher concentration cases point to energy being diminished in this region due to increased particle interaction.

Reynolds shear stress within the near wake is most drastically different below hub height between the sub-inertial and particle concentration cases, representing decrease in turbulent energy contributions with the presence of particles. The dominant peak for all cases is present at rotor bottom height. Compared to the axisymmetric wakes observed in previous turbine studies, all near wake profiles show significant magnitudes in the lower portion of the turbine shadow. This is assumed to be due to interactions of the flow with the tower. In the far wake, all $\Phi_{v}$ cases appear to collapse, representing the symmetric behavior as discussed in previous literature [38].

While vertical fluctuation contributions to kinetic energy $\left(\overline{v^{\prime} v^{\prime}} / U_{\infty}\right)$ share similarity in the near wake for all $\Phi_{v}$ cases, and collapse above $y / D=0.2$, the presence of inertial particles has an apparent diminishing effect compared to $\Phi_{0}$ within the rotor wake. As particles interact with the turbine model, they are less likely to contribute to energy content within the wake overall. In the far wake region, small differences between the smoke and inertial particle cases are visible within the rotor profile, but relatively small contributions are observed by all scenarios. Connecting with the previous quantities, energy contributions from streamwise fluctuations dominate within the turbine wake. 


\subsection{Voronoi Analysis}

Voronoi analysis was performed within the far wake region of the turbine model to observe clustering behavior. Figure 4.7 contains visual and statistical Voronoi tessellation results for a single representative snapshot in the far wake region with inflow of $R e_{\lambda}=66.58$ and particle concentration of $\Phi_{1.7}$. The same procedure was performed for several independent realisations to confirm consistency of results.

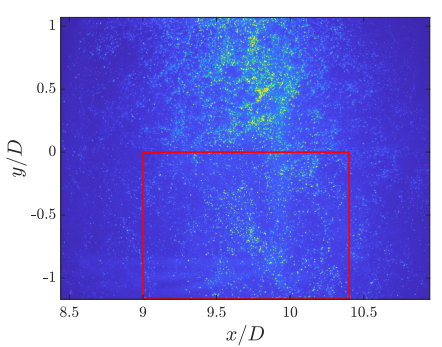

(A)

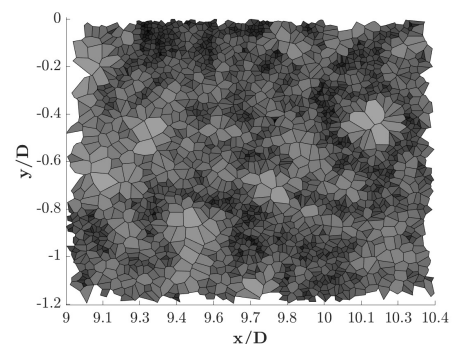

(B)

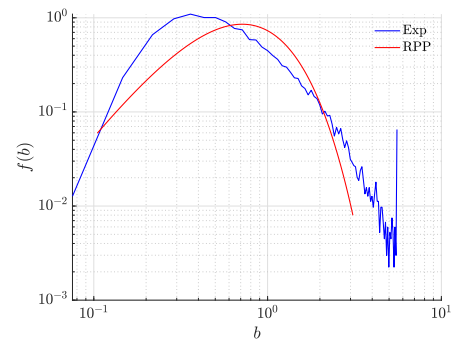

(C)

Figure 4.7: Voronoi analysis images for $R e_{\lambda}=66.58, \Phi_{\nu}=\Phi_{1.7}$ : (a) Sample PIV image used for particle detection, interrogation area outlined in red; (b) Voronoi cell image for a single image; (c) PDF of normalized cell area $b=S /\langle S\rangle$ plotted against random Poisson process distribution.

The image in Figure 4.7(A) is the colored representation of a single PIV snapshot used to calculate Voronoi quantities, where the red rectangle represents the interrogation region for Voronoi tessellation. Cell centers were defined by locating image pixels of a specified intensity range to consider particles within the same planar distance from the camera location. The resulting center points are then used to construct Voronoi cells based on the perpendicular bisect method, creating polygonal regions as shown in Figure 4.7(B), with 
shape and areas defined by lines of proximity to nearest surrounding neighbors. Cells are presented in grayscale to emphasize area differences. Small, darker cells represent particle clustering regions and larger, lighter cells are voids. Dark clustering regions are observed surrounding the voids, implying locations of turbulent scale features. Probability density functions $f(b)$ versus normalized cell area $b=S /\langle S\rangle$ are shown for both the experimental image (Exp) and the random Poisson process (RPP) in Figure 4.7(C), where $S /\langle S\rangle$ is the normalized cell area for the region. The standard deviation of the experimental distribution for the shown case is $\sigma_{\text {voro }}=0.91$, aligning with previous theory as higher than that of the random process $\sigma_{R P P}=0.54$. Additionally, the standard deviation of the open-tunnel distribution was $\sigma_{\text {voro,open }}=0.82$, within the range observed by Monchaux et al [27]. The two points of intersection on the right hand side of this plot are referred to as $b_{c}$ and $b_{v}$ and their appearance suggests the presence of clusters and voids. Clustering is said to occur for all normalized areas less than $b=b_{c}$, and void regions exist for all greater than $b=b_{\nu}$. The third crossing visible for low values of $b$ does not relate to clustering or void behavior. Rather this intersection speaks to variations in experimental conditions and lack of convergence due to limited data which should be examined for future study [27, 30]. This study does not fully investigate Voronoi analysis, as it would also allow to characterise the size and distribution of clusters and voids. Such discussion is out of the scope of the present work, but these initial results suggest that the method is viable in application to future wind turbine studies. 


\section{Chapter 5}

\section{Conclusions}

This study presents experimental analysis on the wake of a model wind turbine subjected to turbulent, polydispersed, multi-phase flow. Wind tunnel experiments consider three varied carrier wind velocities with water droplets uniformly dispersed into the flow at three varied injection rates from a spray grid at the tunnel entrance for comparison with a buoyant subinertial flow field. Turbulence effects and particle behavior compare single-phase to multiphase flow within the near and far wake of the model using hot wire anemometry, phase doppler interferometry (PDI), and particle image velocimetry (PIV) data.

Analysis of hot wire measurements at hub height for the single-phase flow case show higher energy content within the wake and increased inflow velocity, with the near wake surpassing that of the far wake and open tunnel configurations for a given $R e_{\lambda}$. Inflow velocities of $2.6,4.86$, and $8.3 \mathrm{~ms}^{-1}$ produce streamwise velocity deficits in the far wake of $25 \%, 18 \%$ and $15 \%$, suggesting wake persistence even at the location of $9.6 \mathrm{D}$ downstream of the model.

Data taken in the near wake of multi-phase flow with PDI show the greatest differences in particle diameter and highest velocity deficits versus the far wake when compared to open-tunnel configurations. The smaller particles within the near wake suggest entrainment of ambient flow and particles into the region. Velocity within the wake is dependent on both inflow carrier velocity and injection flow rate, where near wake deficits for $\Phi_{1.7}$ and

$\Phi_{2}$ range from $1.37-2.7 \mathrm{~ms}^{-1}$ and $1.44-2.84 \mathrm{~ms}^{-1}$ as $R e_{\lambda}$ increases, while far wake deficits range between $0.29-0.74$ and $0.33-1.00 \mathrm{~ms}^{-1}$. For a single inflow velocity at $R e_{\lambda}=66.58$, 
the near wake deficits translate to $59.5 \%$ and $62.62 \%$ losses for $\Phi_{1.7}$ and $\Phi_{2}$ injection rates. Although less prominent, velocity deficits in the far wake are $15.83 \%$ and $19.83 \%$ for the two concentrations which is a significant loss in the context of available flow for subsequent turbines in closely-ordered wind farms.

Contour wake width and velocity profiles show a significant dependence on inflow velocity and particle concentration in PIV analysis. At the lowest inflow velocity, the near wake maintains a similar streamwise velocity profile between the sub-inertial and inertial particle cases. A decrease in wake velocity with the addition of particles versus the subinertial occurs for all inertial flow rates. The moderate and high inflow velocities show greater dependence on injection flow rate, resulting in diverging wakes with larger lowvelocity regions for both near and far wakes. Velocity deficits within the wake core remain between 10-15\% across the far wake for all cases, affirming the lack of wake recovery of a turbine model at this inflow velocity. At the moderate carrier flow of $R e_{\lambda}=66.58$, Vertical velocity profiles show increased downward preference at higher particle concentrations in the near wake than sub-inertial and $\Phi_{1.2}$ particle concentration, specifically at the bottom rotor edge and $0.2 \mathrm{D}$ below hub height. Downward trajectories in the far wake are more pronounced for the moderate concentrations, but all inertial cases ultimately collapse above hub height and emphasize the lingering effect of falling particles due to rotor interaction compared to sub-inertial behavior.

Energy contributions due to streamwise fluctuations $\overline{u^{\prime} u^{\prime}}$ are lower overall for multiphase flow fields versus single phase, yet highlight the effects of particle impact with the model within distinct regions of the rotor shadow. The turbulent momentum flux term $\overline{u^{\prime} v^{\prime}}$ in the near wake maintains a consistent profile for all $\Phi_{v}$ with significant energy content at the bottom edge of the rotor, but appears to diminish from the sub-inertial case with the introduction of particle injection beginning at $\Phi_{1.2}$. As concentration increases to $\Phi_{1.7}$ and $\Phi 2$, the energy increases and more closely mimics that of the sub-inertial case. In the far wake, the turbulent momentum flux is minimal for the sub-inertial measurements 
across the rotor profile compared to that of $\Phi_{1.2}-\Phi_{2}$, where the particle presence increases energy content and persistence within the wake. The highest injected concentration displays the highest magnitudes of $\overline{u^{\prime} v^{\prime}}$ in both near and far wake, demonstrating increases in turbulence production on the continued wake. Momentum contributions due to vertical fluctuations $\overline{v^{\prime} v^{\prime}}$ are similar for sub-inertial and inertial particle cases, yet demonstrate an energy-decreasing effect due to particle presence compared to single-phase flow. This effect persists even into the far wake throughout the rotor profile and represents diminished energy content injected into the wake caused by the particle impact with the turbine.

Voronoi analysis was used as an exploratory exercise to examine validity of the method when applied to particle-laden wakes of turbines. Images captured with PIV data in the model far wake were used to determine pixel-based cell centers and create corresponding areas via the perpendicular bisection method relative to proximity of nearest neighbors. The resulting Voronoi diagrams contain clearly defined regions suggestive of clustering and voids typically found in turbulent flows. The intersection points observed between the probability distribution of cell areas and that of a random process show similarity to those obtained in more exhaustive studies [27, 30]. Although a more focused discussion on cluster and void areas and distributions is necessary for full Voronoi analysis, the validated results presented suggest this method to be useful for defining particle behavior in turbine wakes and inspires further examination. 


\section{Chapter 6}

\section{Future Work}

This new insight into particle behavior in turbine wakes inspires future work toward indepth Voronoi analysis of clustering behavior, further experiments observing effects within multi-unit wind plants, and ultimately employing this analysis to other large-scale renewable energy sources. Application of statistical and spatial Voronoi distribution techniques to turbines in varied multi-phase flow conditions will be employed to more fully consider the relationship of clustering and turbulent wake propagation. Further experimental studies will consider an array of multiple turbines subjected to varied inflows and particle concentrations while observing particle interaction within compounded turbulence. By combining this information with experiments in force loading due to particle impact on turbine blades, the question of whether clustering plays a role in blade fatigue can be examined. These methods will be applied to experiments with other renewable resources, such as solar photovoltaic systems, to explore the effects of multi-phase turbulent flows in terms of particle deposition and destructive forcing on physical components. Through imposition of varied particle densities and concentrations, classification of behavior within climatedependent debris fields can be determined. This information will help inform design of new and existing wind energy harvesting systems toward damage mitigation and overall efficiency. 


\section{Bibliography}

[1] BP Statistical Review of World Energy. Technical report, BP p.l.c., 2019.

[2] U.S. Wind Industry Quarterly Market Report, Fourth Quarter 2019. Technical report, American Wind Energy Association, 2019. Online; Accessed: 2020-04-30.

[3] U. E. I. Administration. How much electricity does an american home use? Online; Accessed: 2020-04-30.

[4] J. Bec, L. Biferale, M. Cencini, A. Lanotte, S. Musacchio, and F. Toschi. Heavy Particle Concentration in Turbulence at Dissipative and Inertial Scales. Phys. Rev. Lett., 98(8):084502, Feb. 2007.

[5] T. Burton, N. Jenkins, D. Sharpe, and E. Bossanyi. Wind Energy Handbook. John Wiley \& Sons, Inc., 2 edition, 2011.

[6] M. Cai, E. Abbasi, and H. Arastoopour. Analysis of the Performance of a WindTurbine Airfoil under Heavy-Rain Conditions Using a Multiphase Computational Fluid Dynamics Approach. Ind. Eng. Chem. Res., 52(9):3266-3275, Mar. 2013.

[7] R. B. Cal and M. Gibson. Statistical characteristics of a $3 \times 3$ neutrally stratified wind turbine array boundary layer. Progress in Turbulence and Wind Energy IV, 141, 2012.

[8] L. P. Chamorro and F. Porté-Agel. A Wind-Tunnel Investigation of WindTurbine Wakes: Boundary-Layer Turbulence Effects. Boundary-Layer Meteorology, 132(1):129-149, July 2009.

[9] A. C. Cohan and H. Arastoopour. Numerical simulation and analysis of the effect of rain and surface property on wind-turbine airfoil performance. International Journal of Multiphase Flow, 81:46-53, May 2016.

[10] R. D. Corrigan and R. D. DeMiglio. Effect of Precipitation on Wind Turbine Performance. Technical report, National Aeronautics and Space Administration, May 1985.

[11] T. Dairay, M. Obligado, and J. C. Vassilicos. Non-equilibrium scaling laws in axisymmetric turbulent wakes. Journal of Fluid Mechanics, 781:166-195, Oct. 2015.

[12] E. Dunham. The potential influence of rain on airfoil performance. von Karman Institue for Fluid Dynamics, Feb. 1987. 
[13] J. Eaton and J. Fessler. Preferential Concentration of Particles By Turbulence. International Journal of Multiphase Flow, 20:169-209, 1994.

[14] B. C. W. Farm. Blue canyon wind farm gallery. Online; Accessed: 2020-04-30.

[15] J.-S. Ferenc and Z. Néda. On the size distribution of Poisson Voronoi cells. Physica A: Statistical Mechanics and its Applications, 385(2):518-526, Nov. 2007.

[16] G. Fiore and M. S. Selig. Optimization of Wind Turbine Airfoils Subject to Particle Erosion. American Institute of Aeronautics and Astronautics, June 2015.

[17] G. Fiore and M. S. Selig. Simulation of Damage Progression on Wind Turbine Blades Subject to Particle Erosion. American Institute of Aeronautics and Astronautics, Jan. 2016.

[18] G. Good, P. Ireland, G. Bewley, E. Bodenschatz, L. Collins, and Z. Warhaft. Settling regimes of inertial particles in isotropic turbulence. Journal of Fluid Mechanics, 759, Nov. 2014.

[19] S. Goto and J. C. Vassilicos. Sweep-Stick Mechanism of Heavy Particle Clustering in Fluid Turbulence. Phys. Rev. Lett., 100(5):054503, Feb. 2008.

[20] N. Hamilton, M. Melius, and R. B. Cal. Wind turbine boundary layer arrays for Cartesian and staggered configurations- Part I , flow field and power measurements: Wind turbine arrays in Cartesian and staggered configurations. Wind Energy, 18(2):277295, Feb. 2015.

[21] K. S. Hansen, R. Barthelmie, L. E. Jensen, and A. Sommer. The impact of turbulence intensity and atmospheric stability on power deficits due to wind turbine wakes at horns rev wind farm. Wind Energy, 15(1):183-196, 2012.

[22] H. Homann and J. Bec. Concentrations of inertial particles in the turbulent wake of an immobile sphere. Phys. Fluids, 27(5):053301, May 2015.

[23] M. G. Khalfallah and A. M. Koliub. Effect of dust on the performance of wind turbines. Desalination, 209(1-3):209-220, Apr. 2007.

[24] D. Li, Z. Zhao, Y. Li, Q. Wang, R. Li, and Y. Li. Effects of the particle Stokes number on wind turbine airfoil erosion. Appl. Math. Mech.-Engl. Ed., 39(5):639-652, May 2018.

[25] M. Massee. Dust storm through wind farms, mojave, ca., 2012. Online; Accessed: 2020-04-22.

[26] J. Menkes, R. Sreedhar, and C. Hindman. Entrainment in turbulent wake flows. Phys. Fluids, 17(1):37, 1974.

[27] R. Monchaux, M. Bourgoin, and A. Cartellier. Preferential concentration of heavy particles: A Voronoï analysis. Phys. Fluids, 22(10):103304, Oct. 2010. 
[28] R. Monchaux, M. Bourgoin, and A. Cartellier. Analyzing preferential concentration and clustering of inertial particles in turbulence. International Journal of Multiphase Flow, 40:1-18, Apr. 2012.

[29] D. O. Mora, E. M. Pladellorens, P. R. Turró, M. Lagauzere, and M. Obligado. Energy cascades in active-grid-generated turbulent flows. Phys. Rev. Fluids, 4(10):104601, Oct. 2019.

[30] M. Obligado, A. Cartellier, A. Aliseda, T. Calmant, and N. de Palma. Study on preferential concentration of inertial particles in homogeneous isotropic turbulence via big-data techniques. Phys. Rev. Fluids, 5:024303, Feb 2020.

[31] Y. Odemark and J. H. M. Fransson. The stability and development of tip and root vortices behind a model wind turbine. Exp Fluids, 54(9):1591, Sept. 2013.

[32] B. Ofner. Phase Doppler Anemometry (PDA). In F. Mayinger and O. Feldmann, editors, Optical Measurements: Techniques and Applications, pages 139-152. Springer Berlin Heidelberg, 2001.

[33] S. Pol, A. Taylor, A. Bilbao, A. Doostalab, S. Novoa, C. Westergaard, F. Hussain, J. Sheng, B. Ren, M. Giesselmann, M. Glauser, and L. Castillo. Field measurements in the wake of a model wind turbine. Journal of Physics: Conference Series, 524, 2014.

[34] S. Sumbekova, A. Cartellier, A. Aliseda, and M. Bourgoin. Preferential concentration of inertial sub-kolmogorov particles. The roles of mass loading of particles, Stokes and Reynolds numbers. Phys. Rev. Fluids, 2(2):024302, Feb. 2017.

[35] F. Toschi and E. Bodenschatz. Lagrangian Properties of Particles in Turbulence. Annu. Rev. Fluid Mech., 41(1):375-404, Jan. 2009.

[36] S. L. Tours. May 4th adrian, texas tornado. Online; Accessed: 2020-04-30.

[37] L. Vermeer, J. Sørensen, and A. Crespo. Wind turbine wake aerodynamics. Progress in Aerospace Sciences, 39(6-7):467-510, Aug. 2003.

[38] K. Viestenz and R. B. Cal. Streamwise Evolution of Statistical Events in a Model Wind-Turbine Array. Boundary-Layer Meteorology, 158(2):209-227, Feb. 2016.

[39] S. N. Walker and J. E. Wade. Effects of Precipitation on Wind Turbine Performance. Technical report, Solar Energy Research Institute, Mar. 1988.

[40] W. Zhang, C. D. Markfort, and F. Porté-Agel. Near-wake flow structure downwind of a wind turbine in a turbulent boundary layer. Experiments in Fluids, 52(5):12191235, May 2012. 